\title{
Altimetry-Based Diagnosis of Deep-Reaching Sub-Mesoscale Ocean Fronts
}

\author{
Lia Siegelman ${ }^{1,2, *} \mathbb{( D}$, Patrice Klein ${ }^{1,2,3,4} \mathbb{C}$, Andrew F. Thompson ${ }^{1} \mathbb{D}$ and Hector S. Torres ${ }^{2}(\mathbb{D}$ \\ and Dimitris Menemenlis 2 (D) \\ 1 Environmental Science and Engineering, California Institute of Technology, Pasadena, CA 91125, USA; \\ pklein@caltech.edu (P.K.); andrewt@caltech.edu (A.F.T.) \\ 2 Jet Propulsion Laboratory, California Institute of Technology, Pasadena, CA 91109, USA; \\ Hector.Torres.Gutierrez@jpl.nasa.gov (H.S.T.); dimitris.menemenlis@jpl.nasa.gov (D.M.) \\ 3 Laboratory for Ocean Physics and Satellite remote sensing, Ifremer, CNRS, Univ. de Bretagne Occidentale, \\ 29280 Plouzane, France \\ 4 Laboratoire de Meteorologie Dynamique, Ecole Normale Superieure, CNRS, 75005 Paris, France \\ * Correspondence: 1siegelman@caltech.edu
}

Received: 10 August 2020; Accepted: 25 August 2020; Published: 28 August 2020

\begin{abstract}
Recent studies demonstrate that energetic sub-mesoscale fronts (10-50 km width) extend in the ocean interior, driving large vertical velocities and associated fluxes. However, diagnosing the dynamics of these deep-reaching fronts from in situ observations remains challenging because of the lack of information on the 3-D structure of the horizontal velocity. Here, a realistic numerical simulation in the Antarctic Circumpolar Current (ACC) is used to study the dynamics of submesocale fronts in relation to velocity gradients, responsible for the formation of these fronts. Results highlight that the stirring properties of the flow at depth, which are related to the velocity gradients, can be inferred from finite-size Lyapunov exponent (FSLE) at the surface. Satellite altimetry observations of FSLE and velocity gradients are then used in combination with recent in situ observations collected by an elephant seal in the ACC to reconstruct frontal dynamics and their associated vertical velocities down to $500 \mathrm{~m}$. The approach proposed here is well suited for the analysis of sub-mesoscale-resolving datasets and the design of future sub-mesoscale field campaigns.
\end{abstract}

Keywords: altimetry; finite-size Lyapunov exponent; ocean dynamics; sub-mesoscale

\section{Introduction}

Recent observational and numerical studies revealed the existence of energetic sub-mesoscale fronts (10-50 km width) in regions of elevated Eddy Kinetic Energy (EKE), not only within the surface mixed layer but also below it down to $900 \mathrm{~m}$ [1-4]. These fronts are characterized by Richardson and Rossby numbers of order one, emphasizing their ageostrophic character $[1,3,4]$. They result from the horizontal stirring of buoyancy anomalies by mesoscale eddies (50-300 km size) and are associated with intense vertical velocities [5]. Their impact on oceanic vertical heat transport is an order of magnitude larger than the one associated with mesoscale eddies [6]. However, inferring the dynamics of these sub-mesoscale fronts requires knowledge of the evolution of the 3-D structure of the horizontal velocity over a few days, which is rarely accessible from high-resolution in situ data. Only the surface 2-D velocity field at moderate resolution provided by satellite altimeter is commonly available.

This study aims to assess the pertinence of using satellite observations to infer the dynamics of deep-reaching sub-mesoscale fronts. The main goal is to provide a proof of concept of the usefulness of altimetry data to diagnose the dynamics of ocean fronts in existing sub-mesoscale-resolving observations, and to help design future in situ experiments focused on sub-mesoscale dynamics. 
To do so, we use outputs from a $1 / 48^{\circ}$-resolution numerical simulation in the Antarctic Circumpolar Current (ACC) during springtime. The model exhibits deep-reaching buoyancy fronts, from the surface down to $500 \mathrm{~m}$, which are characterized by Rossby and Richardson numbers of order one. Numerical results highlight the vertical homogeneity of the horizontal stirring properties of the flow down to $500 \mathrm{~m}$, suggesting that it is possible to infer subsurface dynamics from surface fields. We then explore the use of Finite-Size Lyapunov Exponent (FSLE), indicative of the stirring properties of the flow integrated over a Lagrangian trajectory, to recover the orientation and growth rate of deep-reaching ocean fronts. Based on the numerical results, we then combine FSLE diagnosed from satellite altimetry with in situ observations collected by a southern elephant seal, in a similar region and season as the model, to recover the correct orientation, magnitude and growth rate of deep-reaching sub-mesoscale fronts and their associated vertical velocities.

The numerical model and observations are presented in Section 2. The proof of concept based on the numerical results is developed in Section 3. Frontal dynamics inferred from the analysis of new seal-sampled observations combined with satellite altimetry data are discussed in Section 4. Finally, a discussion is offered in Section 5 .

\section{Materials and Methods}

\subsection{LLC4320 Numerical Simulation}

We use the LLC4320 simulation, which is based on a global, full-depth ocean and sea ice configuration of the Massachusetts Institute of Technology general circulation model (MITgcm) $[7,8]$. It includes tides, has a horizontal resolution of $1 / 48^{\circ}$ and 90 vertical levels. We refer the reader to Appendix A of Siegelman [4] and Torres et al. [9] for a full description of the characteristics of this simulation and its validation with observations. A sub-domain of the ACC, east of the Kerguelen Islands and spanning $86-90^{\circ} \mathrm{E}, 48-52^{\circ} \mathrm{S}$ is analyzed. This domain is one of the highest EKE regions of the ACC and has a thermocline that can be as deep as $600 \mathrm{~m}$, whereas the Mixed Layer Depth (MLD) has a relatively shallow average value of $50 \mathrm{~m}$ and local maxima of $100 \mathrm{~m}$ within cyclonic eddies. The domain is large enough to capture numerous mesoscale eddies and the resolution is sufficiently high to resolve sub-mesoscale features such as elongated fronts and sub-mesoscale vortices (Figure 1). The time period ranges from 27 October to 27 December 2011, i.e., late spring in the Southern Hemisphere.

\section{Finite-Size Lyapunov Exponent}

Finite-Size Lyapunov Exponent (FSLE) is a Lagrangian diagnostic that measures the separation of a pair of initially close particles $(\delta \mathbf{X}(t)$, with $\mathbf{X}(t)$ the position of a particle at time $t)$ embedded in a given flow field. The separation's growth rate is given by the FSLE's largest eigenvalue, defined as

$$
\lambda\left(d_{0}, d_{f}\right)=\frac{1}{\tau} \log \left(\frac{d_{f}}{d_{0}}\right)
$$

where $d_{0}\left(d_{f}\right)$ is the initial (final) separation distance and $\tau$ the first time at which a separation $d_{f}$ is reached. $\lambda$ has the dimension of time ${ }^{-1}$. FSLE's eigenvector associated with $\lambda$ gives the orientation of the particles' separation. Large $\lambda$ indicate regions of strong stretching [10]. Noteote that FSLE also characterize the growth rate and orientation of lateral gradients of buoyancy [11-13] (see Appendix A for an explanation).

FSLE are computed hourly from the model full velocity field and from the model velocity field after application of a low-pass filter with a cutoff wavelength of $20 \mathrm{~km}$ (Appendix C). We choose $d_{0}=0.01^{\circ}$ and $d_{f}=0.2^{\circ}$ to capture mesoscale and sub-mesoscale features [10]. FSLE are computed backward in time (negative values) using the code provided by AVISO and available at https:/ / bitbucket.org/cnes_aviso/lagrangian/src/master/. 

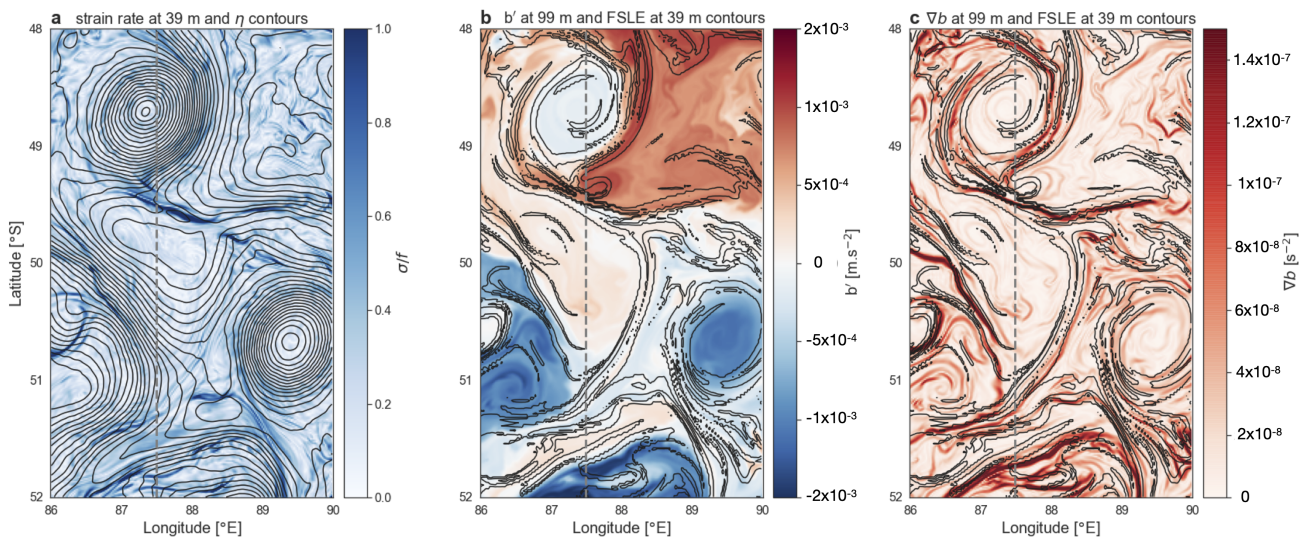

Figure 1. Map of (a) strain rate $\sigma$ normalized by $f$ at $39 \mathrm{~m}$ superimposed with contours of $\eta$ ranging from -1.85 to $-0.85 \mathrm{~m}$ at $0.03 \mathrm{~m}$ interval. (b) Buoyancy anomaly $\mathrm{b}^{\prime}$, defined as the anomaly with respect to the domain-averaged value, at $99 \mathrm{~m}$ superimposed with contours of finite-size Lyapunov exponent (FSLE) at $39 \mathrm{~m}$ ranging from -2.25 to -0.4 day $^{-1}$ at 0.5 day $^{-1}$ interval. (c) Buoyancy gradients $|\nabla b|$ at $99 \mathrm{~m}$ superimposed with the same FSLE contours as in (b). The dashed gray line at $87.5^{\circ} \mathrm{E}$ corresponds to the section in Figure 2. Randomly selected snapshot on 27 October 2011 00:00:00 UTC.
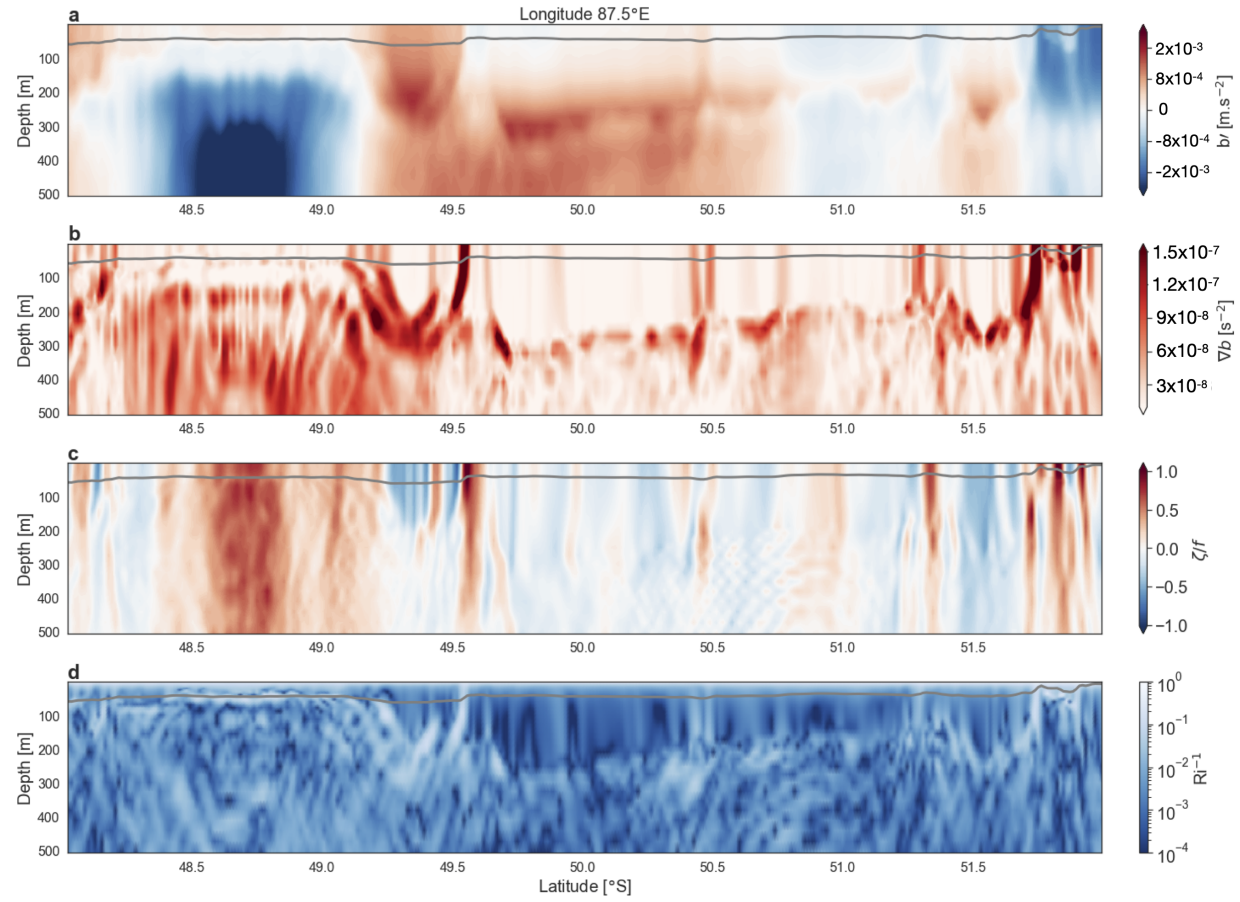

Figure 2. Vertical/meridional section taken at a longitude of $87.5^{\circ} \mathrm{E}$ (dashed gray line in Figure 1) of (a) buoyancy anomaly $b^{\prime}$. (b) Buoyancy gradient $|\nabla b|$. (c) Rossby number $\zeta / f$. (d) Inverse Richardson number $\mathrm{Ri}^{-1}$. The MLD is shown in gray and corresponds to a density increase of $0.03 \mathrm{~kg} \mathrm{~m}^{-3}$ from the density at $10 \mathrm{~m}$. Randomly selected snapshot on 27 October 2011 00:00:00 UTC.

\subsection{Altimetry Data}

We use the Ssalto/Duacs $1 / 4^{\circ}$ delayed-time L4 gridded Sea Surface Height and derived variables provided by Copernicus Marine and Environment Monitoring Service from 6 November to 15 November 2018. The data is available at http://marine.copernicus.eu/services-portfolio/accessto-products/. Vorticity, strain rate and the Okubo-Weiss quantity are computed each day from the altimetry-derived geostrophic velocities.

We use the $1 / 25^{\circ}$ delayed-time FSLE value-added product provided by AVISO from 6 November to 15 November 2018. FSLE are computed backward with $d_{0}=0.02^{\circ}$ and $d_{f}=0.6^{\circ}$. The data is 
available at https://www.aviso.altimetry.fr/es/data/products/value-added-products/fsle-finitesize-lyapunov-exponents.html.

Lagrangian time series of geostrophic velocities and FSLE's orientation are extracted along the seal's path.

\subsection{Southern Elephant Seal Dataset}

We analyze a new in situ dataset collected by a female southern elephant seal from October 2018 to January 2019 east of the Kerguelen Islands in the ACC. We refer the reader to the Methods in Siegelman et al. [3] for more details on the data and correction procedure. However, briefly, the seal was equipped with a CTD-SRDL (CTD-Satellite Relay Data Logger) tag recording conductivity, temperature and pressure at a frequency of $0.5 \mathrm{~Hz}$, subsequently interpolated onto a vertical grid of $1 \mathrm{~m}$ resolution. The transect of interest spans $500 \mathrm{~km}$ between 6 November and 15 November 2018 with a median spacing between two dives of $800 \mathrm{~m}$, and is further discussed in Section 4 . In this transect, the maximal depth of a dive is $800 \mathrm{~m}$ and more than $90 \%$ of the dives are deeper than $200 \mathrm{~m}, 50 \%$ deeper than $400 \mathrm{~m}$ and $35 \%$ reach $500 \mathrm{~m}$ or more. The data has been post-processed following the procedure developed in Siegelman et al. [14], yielding a final accuracy of $\pm 0.02{ }^{\circ} \mathrm{C}$ for temperature and $\pm 0.03 \mathrm{~g} / \mathrm{kg}$ for salinity. Potential density is calculated from conservative temperature and absolute salinity with the TEOS-10 equation [15]. The data is available at http:/ / www.meop.net/database/meop-databases/meop-smsdatabase-submesosc.html. Ethical guidelines and experimental protocols set by the Ethics Committee of the Institut Polaire Francais Paul-Emile Victor (IPEV) and the Polar Environment were applied.

\subsubsection{Buoyancy}

Buoyancy, defined as $b=g\left(1-\rho / \rho_{0}\right)$, where $g$ is gravity, $\rho$ is potential density, and $\rho_{0}=1025 \mathrm{~kg} \mathrm{~m}^{-3}$, is calculated along the seal's track. Buoyancy was first linearly interpolated along the seal's track onto a regular grid of $100 \mathrm{~m}$ resolution (i.e., the shortest distance between two consecutive dives) and a moving average with a $1 \mathrm{~km}$ window was subsequently applied. As such, the final dataset has a horizontal resolution of $1 \mathrm{~km}$ and a vertical resolution of $1 \mathrm{~m}$. Buoyancy anomalies are coherent over multiple profiles, such that aliasing of the along-track data does not contaminate the data.

\subsubsection{Vertical Velocities}

The omega equation has proved to be powerful to diagnose vertical velocities [16,17]. Here, we use a 2-D quasi-geostrophic (QG) version of it [3]. The rationale is that buoyancy fronts are assumed to be elongated in the along-front direction ( $y$-direction) such that the along-front gradient of buoyancy can be neglected per respect to the cross-front one (in the $x$-direction). This equation reads:

$$
N^{2} w_{x x}+f^{2} w_{z z}=-2\left(u_{x} b_{x}\right)_{x}
$$

where subscripts indicate derivatives, $x$ is the cross-front direction, $w$ is the vertical velocity field, $u$ the cross-front horizontal velocity, $N$ the mean Brunt-Väisälä frequency that depends only on $z$. Equation (2) is solved using the flexible framework for spectrally solving differential equations provided by Dedalus [18]. The validity of using this version of the omega equation is discussed in the supplementary information of Siegelman et al. [3].

\section{Numerical Results}

\subsection{Physical Context}

The study region is in the ACC, east of the Kerguelen plateau. It is situated in an energetic hot-spot of the ACC in terms of EKE [19]. Multiple mesoscale eddies interact, leading to horizontal velocities of $1 \mathrm{~m} \cdot \mathrm{s}^{-1}$, with a root-mean-square value of $0.3 \mathrm{~m} \cdot \mathrm{s}^{-1}$, as well as strong horizontal velocity gradients. 
Indeed, both the relative vorticity $\zeta=v_{x}-u_{y}$ and strain rate $\sigma=\sqrt{\sigma_{n}+\sigma_{s}}$ with $\sigma_{n}=u_{x}-v_{y}$ the normal strain rate and $\sigma_{s}=v_{x}+u_{y}$ the shear strain rate, are of the order of $f$, with $f$ the Coriolis frequency (Figure 1a). Mesoscale buoyancy anomalies are present throughout the domain (Figure 1b) and are consistent with the thermal wind balance, i.e., positive (negative) within anticyclones (cylcones). Buoyancy anomalies are stretched by the background mesoscale field, creating lateral gradients of buoyancy $(\nabla b)$ on the edges of and in between mesoscale eddies (Figure 1c). Lateral gradients of buoyancy are mostly at sub-mesoscale: they have a width of $\sim 10 \mathrm{~km}$ and are meandering over length-scale of tens of kilometers. $\nabla b^{\prime}$ s magnitude reaches $4 \times 10^{-7} \mathrm{~s}^{-2}$, which corresponds to a Richardson number of order one. The Richardson number is defined as $\mathrm{Ri}=N^{2} /\left(u_{z}^{2}+v_{z}^{2}\right)$ with $N$ the local Brunt-Väisälä frequency and $\mathbf{u}=(u, v)$ the horizontal velocity vector. Rossby and Richardson numbers of order one are indicative of an ageostrophic regime [5].

The ageostrophic character of the ocean interior is apparent on the meridional/vertical section at $87.5^{\circ} \mathrm{E}$ (Figure 2). Figure 2a highlights the vertical structure of the mesoscale eddies [20]: buoyancy anomalies are negative and reversed bowl shaped in cyclonic eddies. The eddy topology is diverse: some eddies are trapped at the surface, others extend well below the mixed layer (gray line in Figure 2), and some are only present at depth. The MLD has an average value of $50 \mathrm{~m}$. Hence, in this study, the properties diagnosed at $39 \mathrm{~m}$ are representative of the mixed layer, including the surface. The depth extension of the eddies varies between $\sim 100 \mathrm{~m}$ to at least $500 \mathrm{~m}$. Intense $\nabla b$ are located at their periphery, both within and below the mixed layer. $\nabla b$ border buoyancy anomalies, consistent with the maps in Figure $1 b, c$ and what is observed in in situ observations, as will be discussed in Section $4 . \nabla b$ are often slanted along buoyancy anomalies, as can be observed between $49-49.5^{\circ} \mathrm{S}$ in Figure $2 \mathrm{~b}$. These slanted $\nabla b$ are known to result from the competition between horizontal strain and vertical shear [21-23]. Lateral gradients of buoyancy are also present at the base of the mixed layer (48.2-49 $\mathrm{S}$ ), likely the signature of Internal Gravity Waves (IGWs), similar to what has been observed in sub-mesoscale-resolving observations $[1,3]$. The strongest $\nabla b$ are associated with a Rossby number of order one (Figure 2c). Anticyclonic eddies are surrounded by rings of $\zeta$ of opposite sign. At depth, diamond patterns of $\zeta / f$ are present (50.5-51 $\mathrm{S}$ in Figure 2c), illustrating the IGWs' impact below the permanent thermocline. The distribution of $\mathrm{Ri}^{-1}$ closely resembles that of $\nabla b$ despite a background stratification characterized by $N / f \sim 60$. Large $\mathrm{Ri}^{-1}$ are collocated with large $\nabla b$ (Figure $2 \mathrm{~d}$ ). These large $\mathrm{Ri}^{-1}(\geq 0.1-0.2)$ further highlight the ageostrophic nature of the ocean interior, known to be conducive to intense frontogenesis.

Furthermore, the wavenumber spectrum of $\nabla b$-amplitude displays a slope shallower than $k^{-1}$ from 39 to $299 \mathrm{~m}$ and steeper below in the spectral band $10^{-2}-10^{-1} \mathrm{cpkm}$ (Figure 3). Quasi-geostrophic (QG) scaling predicts a $k^{-3}$ spectral difference between $\nabla b$-amplitude at the surface and in the interior [24,25]. This is clearly not the case here, highlighting the departure from QG dynamics in the ocean interior, consistent with previous numerical studies $[4,26]$.

Filaments and vortices of relative vorticity $($ or $\mathrm{Ro}=\zeta / f$ ) can be separated with the Okubo-Weiss quantity (Figure 4, Equation (A3) in Appendix A). Indeed, although filaments and vortices may have similar Ro, filaments are characterized by much larger strain $(\sigma)$ that vortices [27] and the Okubo-Weiss quantity is just the difference between $\zeta^{2}$ and $\sigma^{2}$. Using this partition, Roullet and Klein [27] showed that filaments of vorticity have a larger positive skewness than vortices due to ageostrophic frontogenesis. Here, we use the same Okubo-Weiss partitioning as Roullet and Klein [27] to exclude coherent vortices (Figure 4). $|\nabla b|$ and vorticity filaments are positively and asymmetrically correlated down to $506 \mathrm{~m}$. Indeed, cyclonic (anticyclonic) filaments of vorticity are collocated with strong (weak) $|\nabla b|$. This illustrates the ageostrophic character of deep sub-mesoscale ocean fronts considered in this study. 


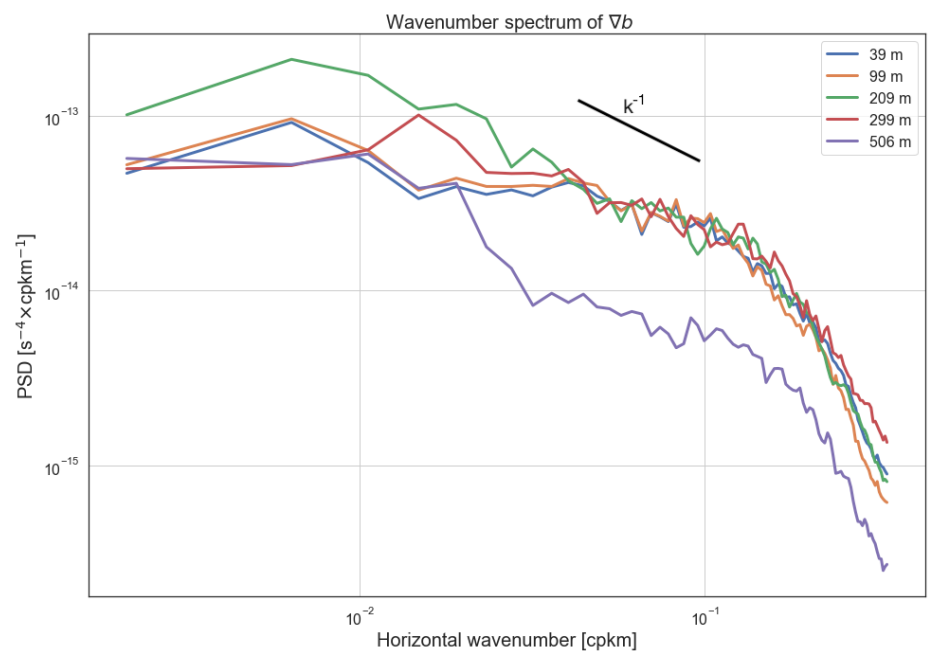

Figure 3. Wavenumber spectrum of $\nabla b$-amplitude at different depths for a randomly selected snapshot taken on 27 October 2011 00:00:00 UTC.
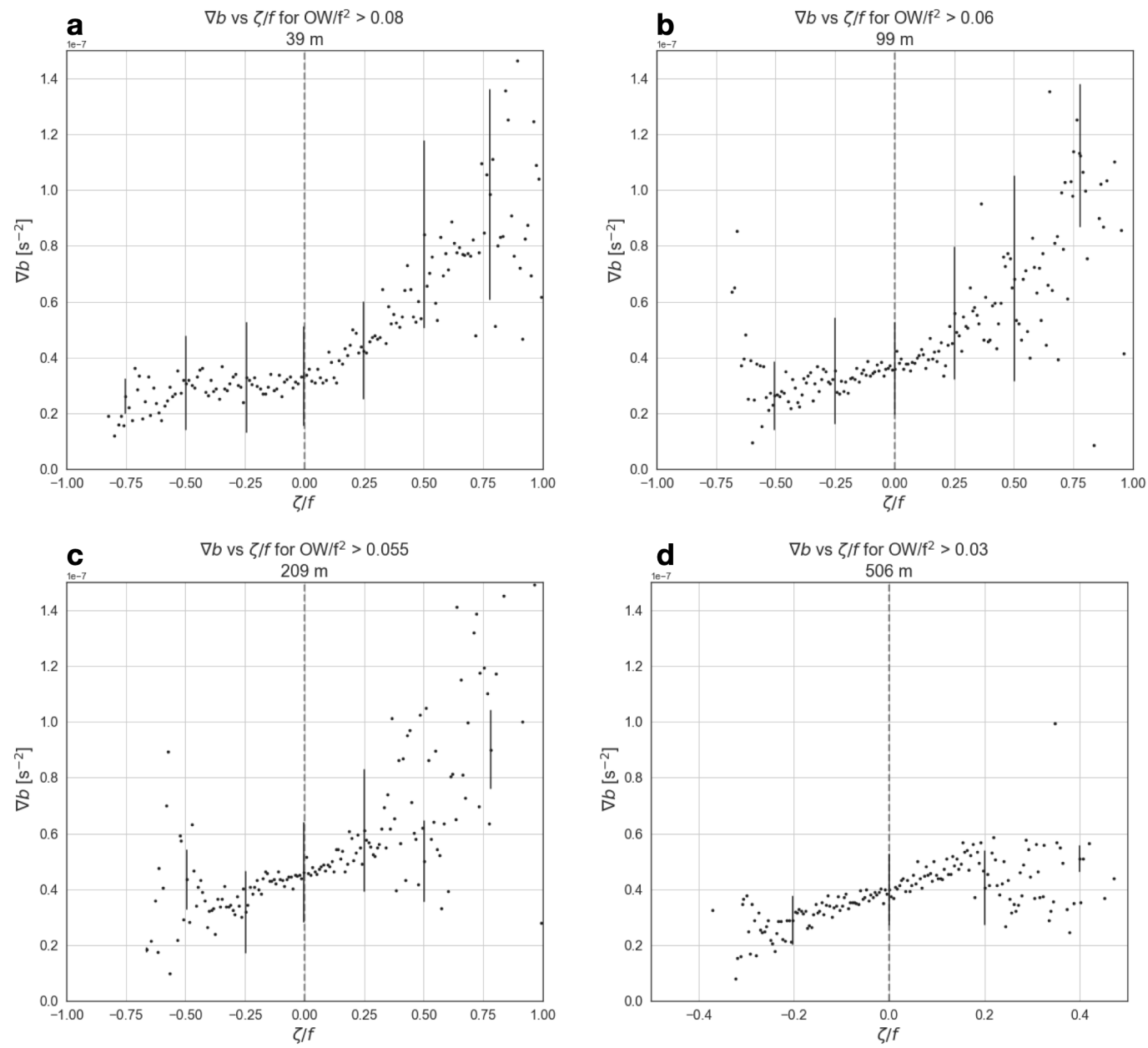

Figure 4. Scatter plots of $|\nabla b|$ and $\zeta / f$ partitioned by Okubo-Weiss $/ f^{2}$ such that $\sim 20 \%$ of the domain points are kept in order to exclude coherent vortices and retain fronts and filaments (see main text, Equation (A3) in Appendix A and Roullet and Klein [27] for more details) at (a) 39 m, (b) 99 m, (c) 209 m, (d) $506 \mathrm{~m}$. Randomly selected snapshot on 27 October 2011 00:00:00 UTC. Dots corresponds to an average over each grid interval on the abscissa (that has a total of 200 grid intervals), and thin vertical lines show std dev around the average. There is a strong asymmetry between positive and negative $\zeta / f$ and $|\nabla b|$, highlighting the ageostrophic regime. 
The simulation includes energetic IGWs, which comprises internal tides, near-inertial motions and a higher frequency IGWs continuum. To distinguish IGWs from balanced motions, i.e., flows in geostrophic or gradient wind balance that comprises meso- and submesoscales [28], we use frequency-wavenumber $(\omega-k)$ spectra (see Appendix B in Siegelman [4] for the methodology). Overall, $\omega-k$ spectra show that strain rate and $|\nabla b|$ are principally explained by scales $\leq 50 \mathrm{~km}$ and that linear IGWs have a weak signature away from the seasonal and permanent thermocline (Figure A2 in Appendix B), emphasizing the existence of deep-reaching energetic frontal dynamics.

\subsection{Frontal Dynamics}

Frontal dynamics are well captured by the equation for the time evolution of a buoyancy gradient, given by [29]:

$$
\frac{d \nabla b}{d t}=-\mathbf{A} \nabla b-N^{2} \nabla w
$$

with $w$ the vertical velocity field, $N^{2}$ the vertical stratification, $\nabla=\left(\partial_{x}, \partial_{y}\right)$ the horizontal gradient operator and $\mathbf{A}$ the velocity gradient tensor, defined as:

$$
\mathbf{A} \equiv \frac{1}{2}\left(\begin{array}{cc}
\sigma_{n} & \sigma_{s}+\zeta \\
\sigma_{s}-\zeta & -\sigma_{n}
\end{array}\right)
$$

with $\zeta$ the relative vorticity, $\sigma_{n}$ the normal strain rate, and $\sigma_{s}$ the shear strain rate (defined in Appendix A) [30]. Equation (3) expresses the balance between the growth of a buoyancy gradient, i.e., $-\mathbf{A} \nabla b$, that destroys the thermal wind balance and the emergence of a vertical velocity field, i.e., $-N^{2} \nabla w$, that acts to restore the thermal wind balance. The first term on the RHS of Equation (3) captures the gradient production by the horizontal velocity gradients, and in particular the strain field. This relation is verified in the model: $\nabla b$-amplitudes are stronger in regions of intense strain (Figure 5).

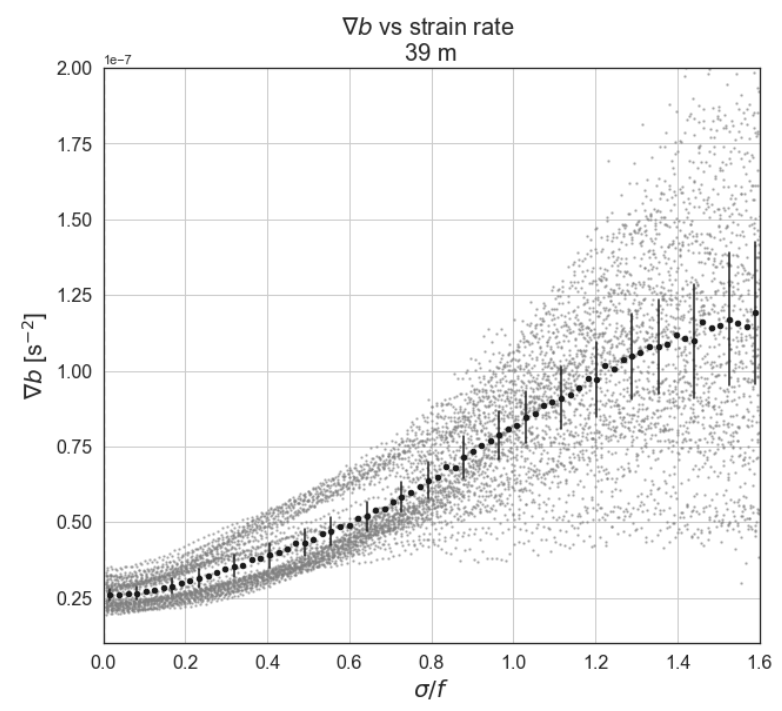

Figure 5. Scatter plot between $|\nabla b|$ and strain rate $\sigma$ normalized by $f$ at 39 m between 27 October and 27 December 2011. Gray points represent the average over each grid interval on the abscissa for a given day (that has a total of 200 grid intervals), black points represents the average over the time period and thin vertical lines show std dev around the average.

Many studies have examined the growth rate and orientation of $\nabla b$ driven by the velocity gradient tensor A (Equation (4)) [30-32]. A brief synthesis of their results is given in Appendix A. In the context of this study, three major results need to be pointed out. First, the orientation of $\nabla b$ at a given depth results from the competition between the strain field at this depth that tends to align $\nabla b$ along the 
compressional strain axis and an "effective" rotation (involving the relative vorticity) at the same depth that tends to remove $\nabla b$ from the compressional axis (see Figure A1 in Appendix A). As a consequence, the orientation of $\nabla b$ may differ from the compressional strain axis and its growth rate may be smaller than the total strain rate $\sigma$ (with $\sigma=\sqrt{\sigma_{n}^{2}+\sigma_{s}^{2}}$ ). Second, the orientation of $\nabla b$ at a given time is not determined by $\mathbf{A}$ at this time but depends on the history of $\mathbf{A}$ over one to a few days. Third, an estimation of the $\nabla b$-growth rate can be recovered from the orientation of $\nabla b$ and the strain rate at this depth. This means that the knowledge of the 3-D evolution of the horizontal velocity gradients over a few days is required to infer the dynamics of sub-mesoscale fronts in the ocean interior. Unfortunately, this complete set of information is rarely accessible from in situ experiments, presenting a major challenge to the observational community.

However, it appears possible to bypass this lack of information using FSLE, in particular those at the ocean surface. First, FSLE at any depth take into account the Lagrangian evolution of the velocity gradient tensor over a few days to estimate the rate and orientation of particles' separation ( $\delta \mathbf{X}$, see Section 2.1). FSLE also characterize the orientation of buoyancy gradients [11-13]. This is because $\nabla b^{T} . \delta \mathbf{X}=\delta b$ is conserved along a Lagrangian trajectory as it is a buoyancy difference between two particles (the buoyancy of each of them being conserved along the trajectory) [11,31]. Second, mesoscale velocities in high EKE regions are usually captured by the first baroclinic mode [33,34]. This implies that FSLE at the surface and at depth should be similar. The accuracy of these two comments is illustrated in Figure 1c, in which FSLE near the surface are aligned with $\nabla b$ at depth (and at the surface, not shown). Based on these comments, the next section explores the relationship between FSLE at different depths and therefore the possibility to infer the dynamics of deep-reaching sub-mesoscale fronts from the observations of $\nabla b$ using FSLE at the ocean surface (as those diagnosed from altimeter data).

\subsection{Recovering Frontal Dynamics at Depth from Finite-Size Lyapunov Exponent at the Surface}

Figure 6 presents FSLE computed from the full model horizontal velocity at different depths. FSLE are elevated around and in between coherent eddies. FSLE filaments are either strongly curved over a small region, typically around sub-mesoscale eddies, or weakly curved over a large region, typically in between mesoscale eddies (Figure 6). This is especially true near the surface (Figure 6a). FSLE associated with sub-mesoscale eddies are shallow and vanish at depth, whereas those associated with mesoscale eddies are deep and persist down to depths of at least $500 \mathrm{~m}$ (Figure $6 \mathrm{~b}-\mathrm{d}$ ), consistent with the fact that energetic eddies are essentially captured by the first baroclinic mode [33,34].
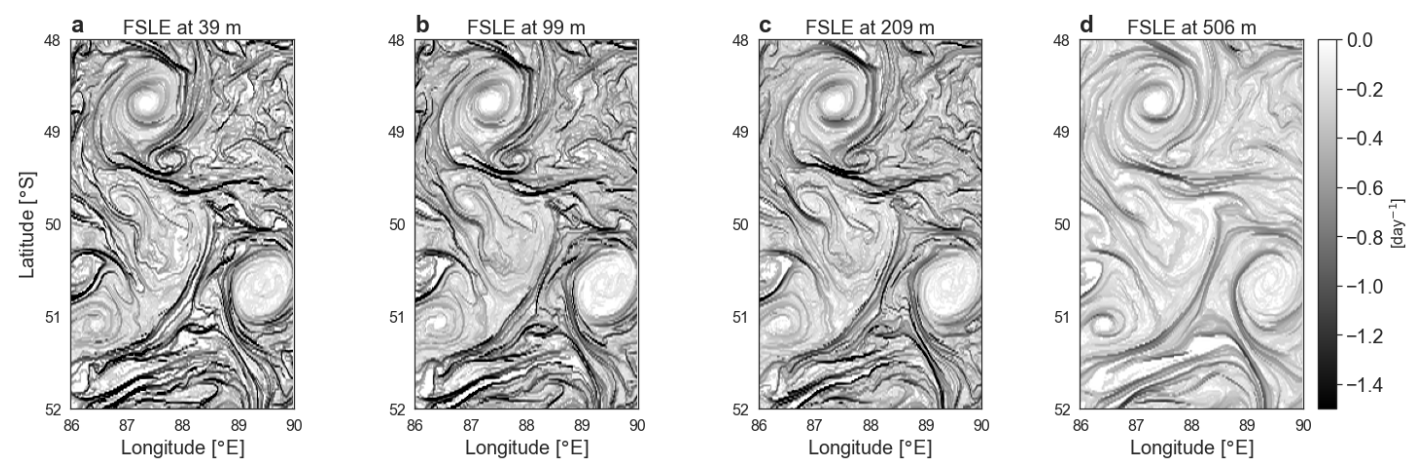

Figure 6. Finite-size Lyapunov exponent (FSLE) computed from the full model velocity field at (a) $39 \mathrm{~m}$, (b) 99m, (c) 209 m, (d) 506 m. Randomly selected snapshot taken on 27 October 2011 00:00:00 UTC. Negative FSLE (computed backward in time [10]) indicate that patches of particles are being stretched and elongated by the background strain field. Large negative FSLE values indicate regions of strong stretching and sub-mesoscale fronts are preferentially located around and in between mesoscale eddies.

A key question that arises is how to identify the part of the strain field that drives the formation of sub-mesoscale buoyancy fronts at the surface and at depth. In other words, is it the mesoscale 
(50-300 km) or the sub-mesoscale $(<50 \mathrm{~km})$ part of the flow that controls the creation of buoyancy fronts? One way to answer this question is to compute the ratio between the strain rate at a given scale and the strain rate at larger scales, as done in Foussard et al. [35]. This ratio can be expressed as:

$$
R_{k}=\frac{\int_{k / 2}^{k} l^{2} E(l) d l}{\int_{k_{\text {min }}}^{k} l^{2} E(l) d l},
$$

with $k$ the wavenumber and $E(k)$ the velocity spectrum. The numerator represents the strain rate associated with eddies of wavenumber $k$ and the denominator captures the strain rate associated with larger eddies [35,36]. Regardless of depth, $R_{k}$ is close to 1 for $k \leq 10^{-2} \mathrm{cpkm}$ but quickly decreases for smaller length-scales (larger $k$ ) and reaches a plateau or a local minimum at length-scales of $\sim 20 \mathrm{~km}$ (Figure 7). This indicates that only scales larger than $20 \mathrm{~km}$ impact the stirring of buoyancy anomalies $[35,36]$.

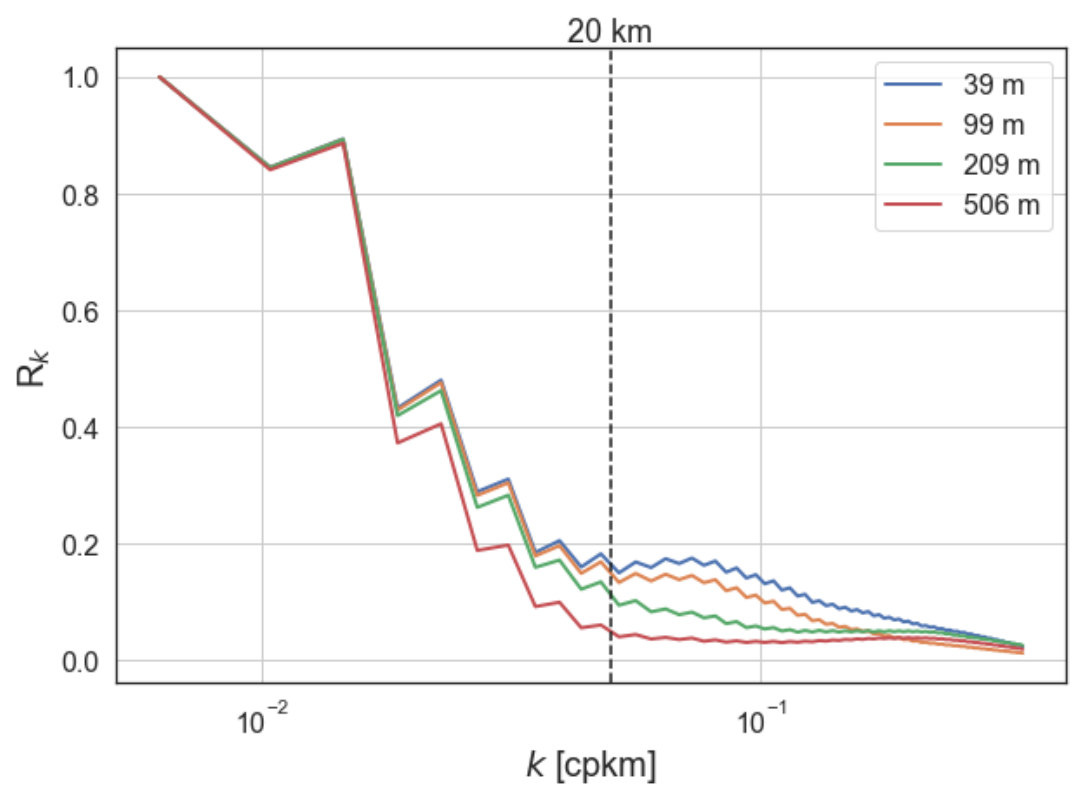

Figure 7. Ratio $R_{k}$ of the strain rate explained by eddies of size $k$ and the strain rate explained by larger eddies. The "effective" strain rate is captured by wavelengths larger than $20 \mathrm{~km}$.

New FSLE are now computed from the model horizontal velocities after application of a low-pass filter with a cutoff wavelength of $20 \mathrm{~km}$ on $u$ and $v$ (see Appendix $C$ for more details on the filter). These FSLE capture the part of the flow that contributes to the stirring of buoyancy anomalies, based on the analysis carried in the previous paragraph (Figure 7). They will exclusively be used in the rest of this section and are referred to as $\mathrm{FSLE}_{\text {filt }}$. FSLE filt look alike at all depths both in terms of magnitude and co-location in physical space (Figure 8a-d). More quantitatively, FSLE filt at $39 \mathrm{~m}$ versus at $209 \mathrm{~m}$ and $506 \mathrm{~m}$ follow a 1-1 relationship (Figure 9). This highlights the good correspondence between FSLE $_{\text {filt }}$ at the surface and at depth, and thus the homogeneity of the stirring properties of the flow down to at least $506 \mathrm{~m}$. A sensitivity analysis indicates that the correlation between FSLE filt $_{\text {near }}$ the surface and at depth decreases when scales smaller than $20 \mathrm{~km}$ are included (not shown). 

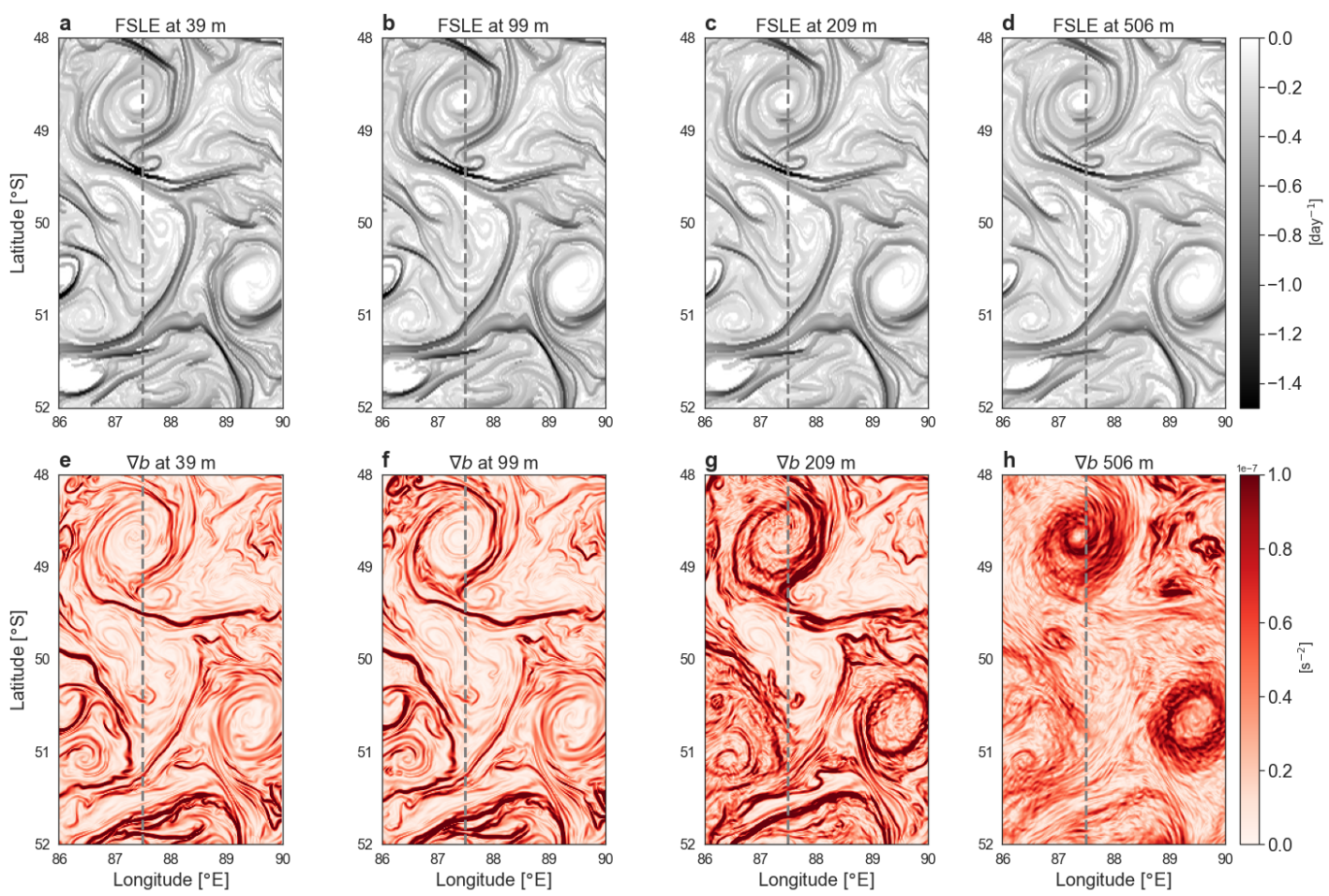

Figure 8. FSLE computed from filtered horizontal velocities (referred to as FSLE $_{\text {filt }}$ in the main text) at (a) $39 \mathrm{~m}$, (b) $99 \mathrm{~m}$, (c) $209 \mathrm{~m}$, (d) $506 \mathrm{~m}$ and $|\nabla b|$ at (e) $39 \mathrm{~m}$, (f) $99 \mathrm{~m}$, (g) $209 \mathrm{~m}$, (h) $506 \mathrm{~m}$. Randomly selected snapshot taken on 27 October 2011 00:00:00 UTC. The dashed gray line corresponds to the sections in Figure 2.
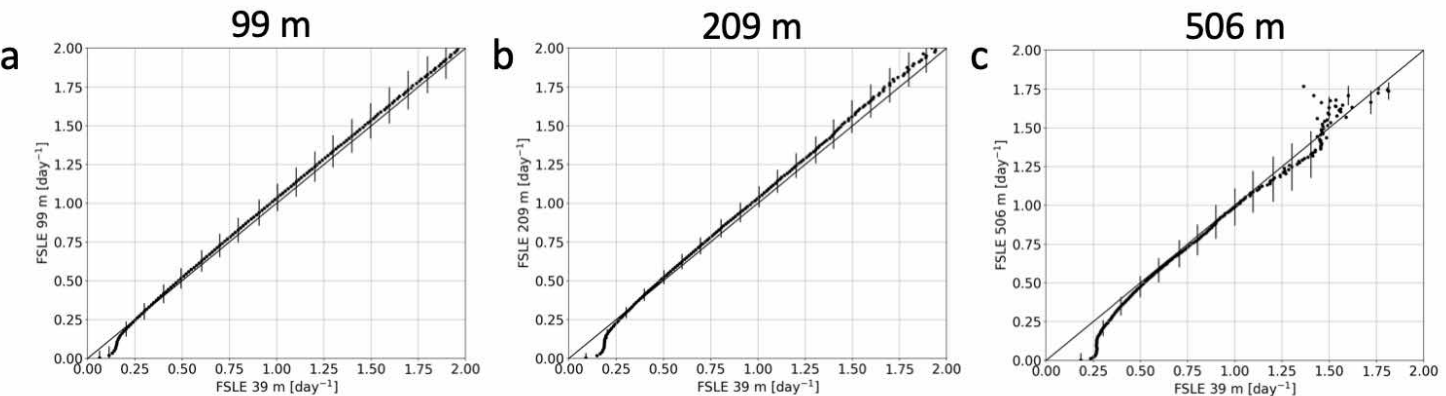

Figure 9. Scatterplot of FSLE filt at $39 \mathrm{~m}$ and FSLE $_{\text {filt }}$ at (a) $99 \mathrm{~m},(\mathbf{b}) 209 \mathrm{~m}$, (c) $506 \mathrm{~m}$ from 27 October to 27 December 2011. The scatterplots are binned on the $y$-axis over 100 bins. FSLE filt $_{\text {at }} 39 \mathrm{~m}$ strongly correlate with FSLE $_{\text {filt }}$ at depth, highlighting the persistence of the vertical structure of the mesoscale strain field down to depths of $506 \mathrm{~m}$. Thin vertical lines show std dev around the average.

Figure 8 (top and bottom panels) reveal the strong similitude between patterns of FLSE $_{\text {filt }}$ and $|\nabla b|$ : both quantities are elongated and/or curved over ten of kms in the same direction. Furthermore, strong $|\nabla b|$ values are almost collocated with strong FSLE filt $_{\text {, }}$ expect at $506 \mathrm{~m}$ where the relationship seems to break down. This may be because at $506 \mathrm{~m}$ (i.e., below the permanent thermocline), IGWs have a signature on $|\nabla b|$ (Figures A2i and 8h) but they are not correlated with the horizontal velocity gradients. A simple reason explaining this regime shift could be that the depth of the permanent thermocline is set by the depth of the winter mixed layer. Figure 10 confirms the overall similitude between patterns of $\operatorname{FLSE}_{\text {filt }}$ and $|\nabla b|$. It emphasizes that $\operatorname{FSLE}_{\text {filt }}$ and $|\nabla b|$ are statistically collocated. Since they are elongated and/or curved over long distances, this result indicates they have statistically the same orientation. These results are consistent with the empirical relation obtained in Siegelman et al. [3], although FSLE from the model have a stronger magnitude than those in Siegelman et al. [3] derived from altimetry. This is due to the different spatial and 
temporal resolution of both velocity fields (hourly and at $1 / 48^{\circ}$ in the model versus daily and at $1 / 4^{\circ}$ in the altimetry product). These findings suggest that strong $|\nabla b|$, both at the surface and in the ocean interior, are preferentially located in regions of intense surface FSLE, i.e., in regions of strong velocity gradients.
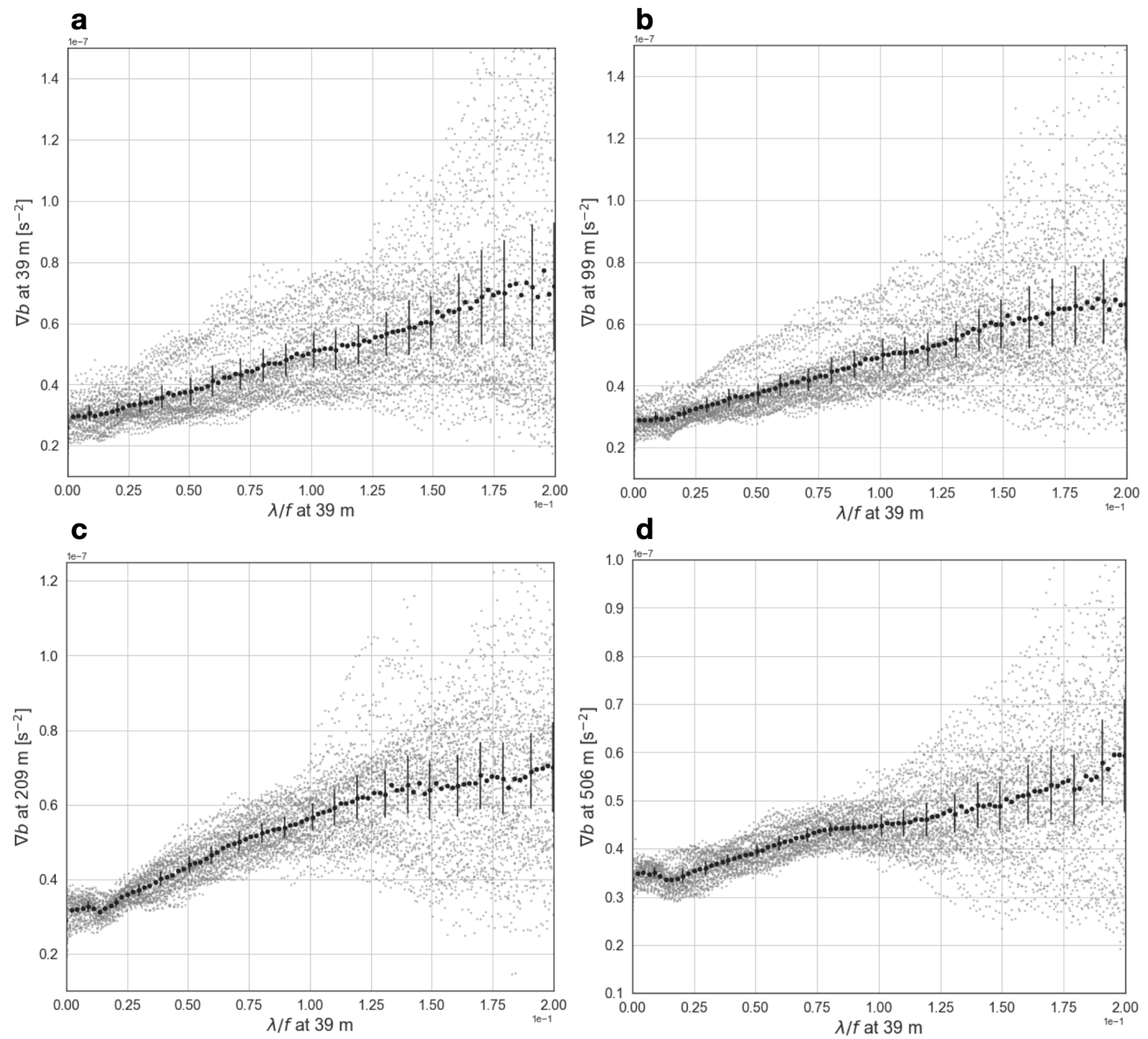

Figure 10. Scatter plots between $\operatorname{FSLE}_{\text {filt }}(\lambda)$ normalized by $f$ at $39 \mathrm{~m}$ and $|\nabla b|$ at (a) $39 \mathrm{~m}$, (b) $99 \mathrm{~m}$, (c) $209 \mathrm{~m}$, (d) $506 \mathrm{~m}$ between 27 October and 27 December 2011. Gray points represent the average over each grid interval on the abscissa for a given day (that has a total of 200 grid intervals), black points represents the average over the time period and thin vertical lines show std dev around the average.

However, one caveat needs to be mentioned. There is a dispersion in the scatter plots of $|\nabla b|$ and FSLE $_{\text {filt }}$ (Figure 10), which can be explained by the phase-lag observed in physical space between the two quantities (Figure 1c). However, even in the presence of a spatial lag, the present results indicate that $\nabla b$ is aligned with its neighboring FSLE $_{\text {filt }}$ (Figures $1 \mathrm{c}$ and 8).

In summary, these numerical results demonstrate that the stirring properties of buoyancy gradients at every depth, i.e., the growth rate and orientation of these gradients (explained by the characteristics of the $3 \mathrm{D}$ velocity gradient tensor $\mathbf{A}$, see Equations (3) and (4)), can be inferred from surface velocity fields in high EKE regions, characterized by $N / f \sim 60$. It suggests that altimetry data can be used to improve the diagnosis of deep-reaching sub-mesoscale dynamics, as discussed in the next section. 


\section{Application to In Situ Data}

Building upon the numerical results of Section 3, we use FSLE and surface geostrophic currents diagnosed from satellite altimetry observations to refine the analysis of sub-mesoscale-resolving in situ observations. The goals of this section are to (i) retrieve the correct orientation, magnitude and growth rate of $\nabla b$ observations, and (ii) derive their associated vertical velocities. Indeed, when a sampling device does not perpendicularly cross a front, it leads to an underestimation of the front's magnitude [1,3].

To achieve (i) and correct for the sampling orientation, the observed buoyancy gradients $b_{x}$, with $x$ the curvilinear abscissa along the instrument's trajectory, are normalized as follows:

$$
b_{x \text { norm }}=\frac{b_{x}}{\sin (|\gamma-\theta|)}
$$

with $\gamma$ the angle between the instrument's trajectory and the $x$-axis of the Cartesian coordinate system and $\theta$ the angle given by the eigenvector associated with the FSLE eigenvalue $\lambda$, which is used to retrieve the orientation of the front with assumptions (see Figure A1 and Appendix A). Equation (6) orients $b_{x \text { norm }}$ in the cross-front direction (with $x$ now the cross-front direction) and adjusts its magnitude. As discussed in the preceding section and in Appendix $\mathrm{A}$, the resulting growth rate of $b_{x \text { norm }}$ is equal to:

$$
\frac{1}{b_{x \text { norm }}} \frac{d b_{x \text { norm }}}{d t}=-u_{x \text { norm }}
$$

with $u_{\text {norm }}$ the cross-front horizontal velocity, $x$ the cross-front direction and $u_{x \text { norm }}$ the cross-front velocity gradient (i.e., $\left.\partial_{x} u_{\text {norm }}\right) . u_{x \text { norm }}$ is estimated from geostrophic surface currents $(u$ and $v$ ) derived from satellite altimetry rotated in the frontal coordinate system by $\theta$ (given by the FSLE's eigenvector, see Appendix A). Note that this method differs from the one developed in Siegelman et al. [3]. Indeed, Siegelman et al. [3] only normalized buoyancy gradients associated with $\lambda>0.15$ day $^{-1}$ and $u_{x \text { norm }}$ was approximated by $\lambda$ along the instrument's trajectory in Equation (7). However, $\lambda$ underestimates the gradients' growth rate [13], which is more accurately given by Equation (7) [11,32,37] (see also Appendix A).

In the frontogenesis framework, the emergence of vertical velocities counterbalances the formation of sharp sub-mesoscale fronts generated by the velocity gradients (Equation (3)). This equilibrium is captured by the classical omega equation [16]. Thanks to the normalization performed in Equation (6), we can achieve (ii) by using a 2-D $(x, z)$ QG version of the omega equation to compute vertical velocities $w$, with $x$ the cross-front direction (see methods for more details on the choice of this equation). We recall the 2-D QG omega equation:

$$
N^{2} w_{x x}+f^{2} w_{z z}=-2\left(u_{x \text { norm }} b_{x \text { norm }}\right)_{x}
$$

with $u_{x \text { norm }}$ the cross-front horizontal velocity gradient, $N$ the mean Brunt-Väisälä frequency that depends only on $z$ and $b_{x \text { norm }}$ the normalized buoyancy gradient. $N$ is estimated from the in situ measurements.

We solve Equations (6) and (8) using a newly available dataset collected by a southern elephant seal east of the Kerguelen plateau in the same season and area as the one studied in Section 3. The transect of interest (in color in Figure 11) has the particularity of sampling several co-interacting mesoscale eddies. As a result, the seal crosses multiple sub-mesoscale fronts that are located at the periphery and in between the mesoscale eddies. The seal's trajectory is often oblique rather than perpendicular to the FSLE's orientation, which makes it an ideal case study to evaluate the impact of our method. 

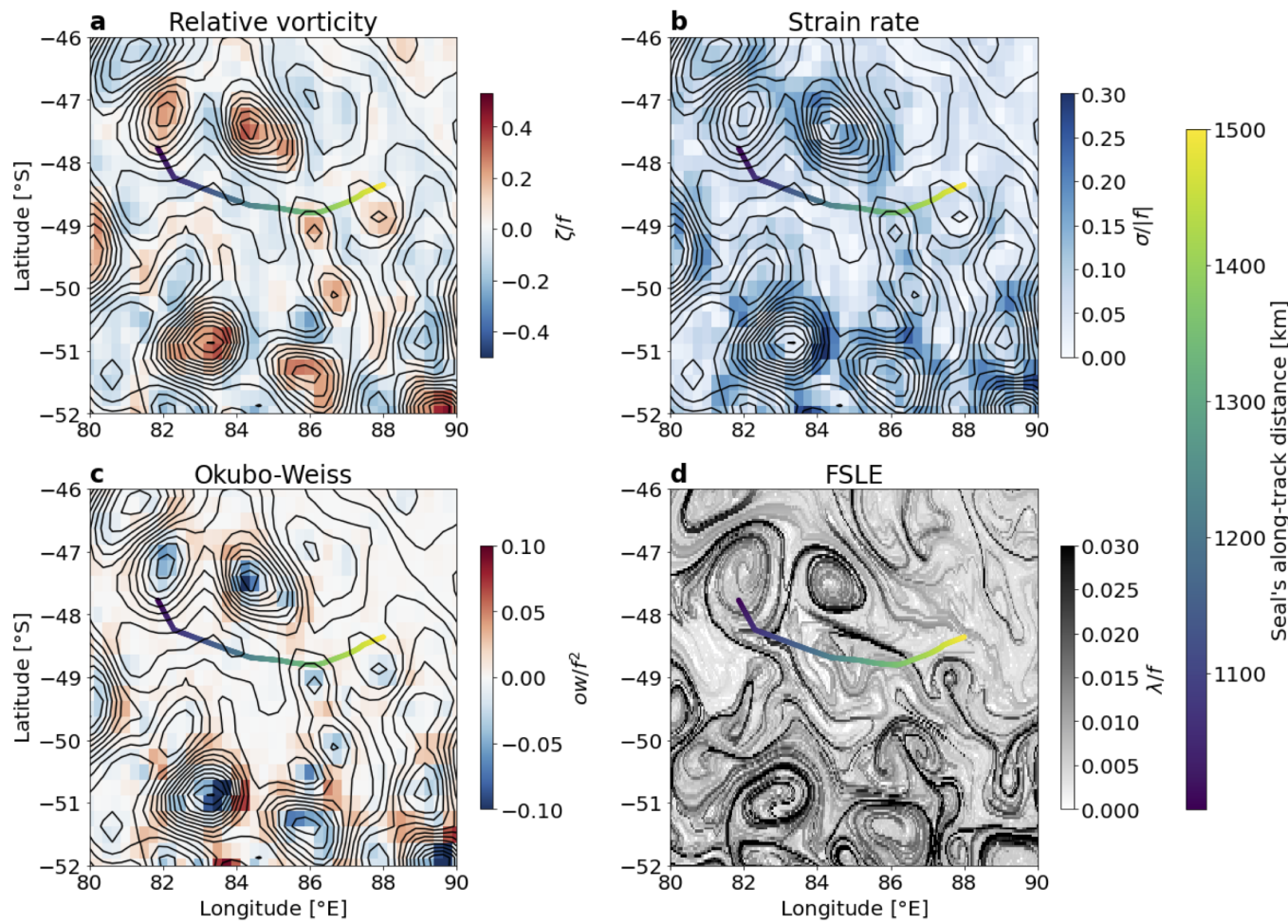

Figure 11. Map of physical quantities derived from AVISO SSH on 11 November 2018 (i.e., at the seal's mid-date trajectory). (a) Relative vorticity normalized by $f$. (b) Strain rate normalized by $f$. (c) Okubo-Weiss quantity normalized by $f^{2}$. (d) FSLE normalized by $f$. The seal's transect is colored by its along-track distance. Note that the mesoscale eddy field considerably evolved during the seal's transect.

At latitudes of $50^{\circ} \mathrm{S}$, Sea Surface Height (SSH) products distributed by AVISO have an effective resolution of $\sim 100 \mathrm{~km}$ [38], i.e., not fine enough to recover the part of the horizontal velocity field in the range $20-100 \mathrm{~km}$ identified in the previous section as being active in the production of frontogenesis-induced $\nabla b$ (Figure 7). Indeed, vorticity, strain rate and the Okubo-Weiss quantity derived from SSH are pixel-like and do not allow the identification of sub-mesoscale fronts and filaments (Figure 11a-c). However, it is possible to recover scales in the $20-100 \mathrm{~km}$ range with the FSLE value-added product distributed by AVISO (Figure 11d). This is because, in the Lagrangian advection framework, fine scales are created through time as a result of the direct cascade of potential energy [11]. FSLE derived from altimetry exhibit filamentary structures that are not present in the other quantities (Figure 11). FSLE's magnitude broadly coincide with the background strain rate's magnitude (Figure $11 b, d$ ). In regions of strong strain, i.e., when the Okubo-Weiss quantity is positive (in red in Figure 11c), FSLE are elevated. Note that as in the model results, FSLE are an order of magnitude lower than strain and vorticity. Hence, from these observations and the theoretical arguments developed in Appendix A, it appears necessary to know both the FSLE's eigenvector and the velocity gradients to recover the orientation and growth rate of buoyancy gradients, respectively.

The impact of the normalization (Equation (6)) is significant (Figure 12). On average, $|\nabla b|$ are 1.8 times larger after normalization and can be as much as 4 times larger (at $1330 \mathrm{~km}$ in Figure 12c). This highlights the usefulness of incorporating the FSLE's orientation into the analysis of sub-mesoscale-resolving datasets. The section sampled by the seal shares a lot of common features with the section from the model (Figure 2). Lateral gradients of buoyancy ( $b_{x}$ norm $)$ are located in between and on the border of mesoscale eddies. They are slanted and follow isopycnals (at 1300-1370 km in Figure 12b) or the MLD (at 1050-1090 km in Figure 12b). $b_{x \text { norm }}$ extend at depth down to at least $500 \mathrm{~m}$, highlighting the presence of energetic submesoscales in the ocean interior. Similar to the model, the signature of IGWs is noticeable at the base of the mixed layer, where $b_{x \text { norm }}$ are organized in 
thin vertical bands of alternating sign (at $\sim 1100 \mathrm{~km}$ in Figure 12b). The inverse Richardson number, defined as $\mathrm{Ri}^{-1}=f^{2} N^{2} / b_{x \text { norm }}^{2}$, assuming thermal wind balance, is of order one at the location of

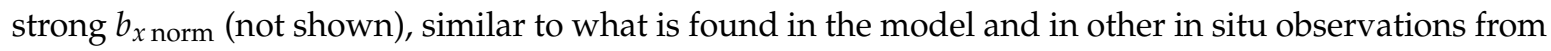
2014 in the same area and season [3]. This suggests an energetic ageostrophic regime associated with an elevated growth rate of buoyancy gradients and intense vertical velocities $w$ [5]. As mentioned above, using the growth rate $u_{x \text { norm }}$ instead of $\lambda$ (as done in Siegelman et al. [3]) makes a considerable difference. Indeed, $\lambda$ consistently underestimates $u_{x \text { norm }}$ (Figure 13), which should then impact the vertical velocities' magnitude, as investigated below.
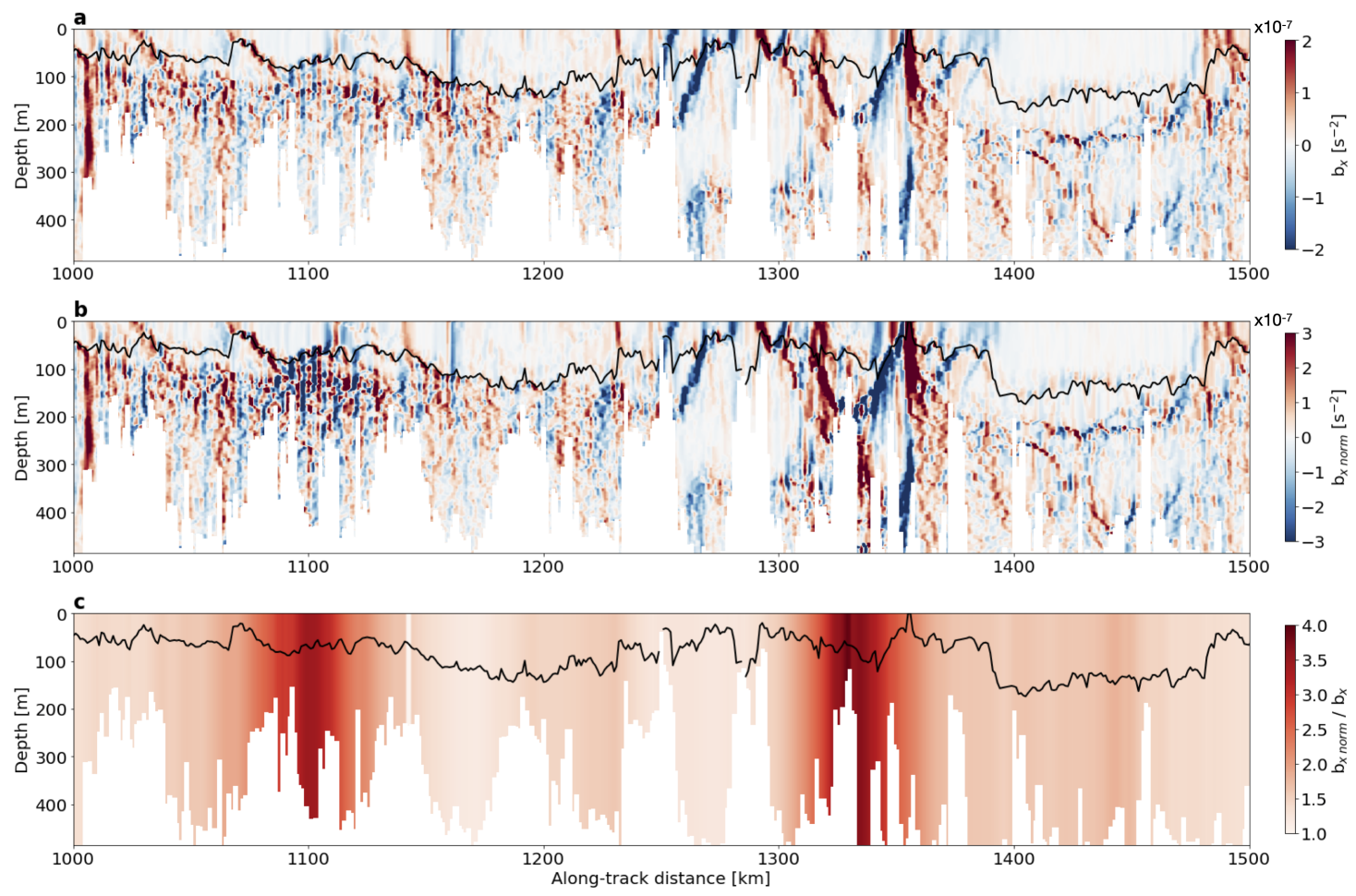

Figure 12. Vertical section of (a) $b_{x}$ sampled by the seal along its trajectory (in color in Figure 11), (b) $b_{x}$ normalized by the angle between the front and the seal's trajectory ( $b_{x \text { norm }}$, see main text and methods), (c) $b_{x \text { norm }} / b_{x}$. The MLD is shown in black and is defined as the level of a $0.03 \mathrm{~kg} \mathrm{~m}^{-3}$ density increase from $15 \mathrm{~m}$ depth.

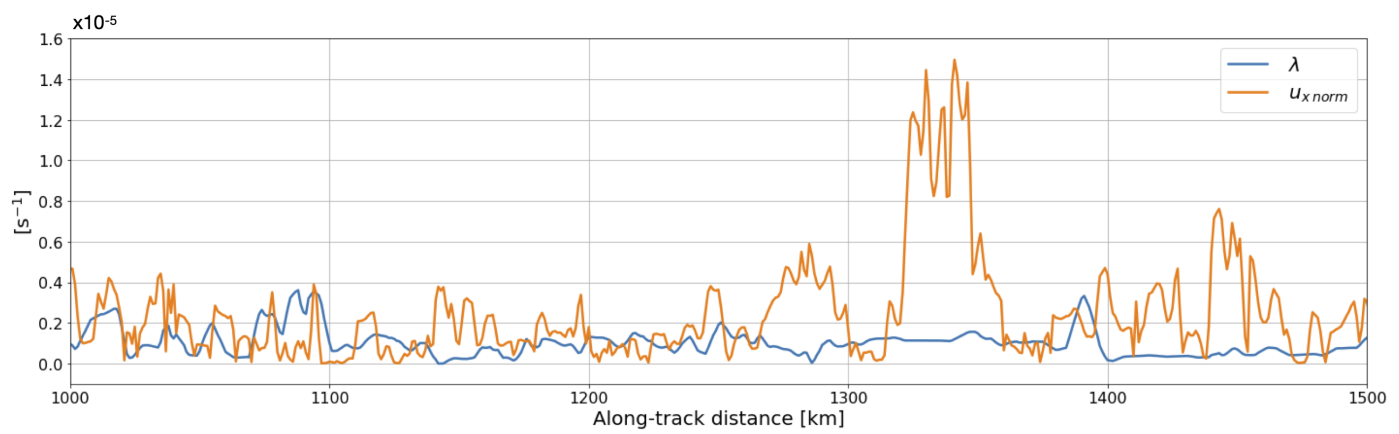

Figure 13. Time series of $\lambda$ (blue curve) and $u_{x}$ norm (orange curve) along the seal's track.

Vertical velocities are presented in Figure 14. In the first panel, Equation (2) is solved in the along-track direction, i.e., with $x$ the curvilinear abscissa along the seal's track. This equation involves $b_{x}$ and $u_{x}$ along the seal's path and yields $w$ (Figure 14a). In the second panel, Equation (8) is now solved in the cross-front direction. This is equivalent to changing the orientation of the fronts along the seal's trajectory such that the fronts are perpendicular to this trajectory (or, equivalently, changing locally 
the seal's trajectory such that this trajectory is perpendicular to the fronts). This equation involves $b_{x \text { norm }}$ and $u_{x \text { norm }}$ cross-front and yields $w_{\text {norm }}$ (Figure $14 \mathrm{~b}$ ). Finally, the third panel shows the ratio between $w_{\text {norm }}$ and $w$ (Figure 14c). Consistent with the buoyancy gradient's normalization (Figure 12), computing vertical velocities in the cross-front direction instead of in the along-track direction makes a significant difference. On average, $w_{\text {norm }}$ is 2.15 times larger than $w$ and can be as much as 15.6 times larger (at $180 \mathrm{~m}$ depth $/ 1335 \mathrm{~km}$ in Figure 14) for $w>20 \mathrm{~m} \mathrm{day}^{-1}$. Even though the sign of the vertical velocity rarely changes between $w_{\text {norm }}$ and $w$, instances where it does (in blue in Figure 14c), as well as instances where $w$ are overestimated compared to $w_{\text {norm }}$ (i.e., when $0<w_{\text {norm }} / w<1$ ), further highlight the importance of considering the horizontal velocity field in the frontal coordinate system. The vertical section of $w_{\text {norm }}$ reveals positive and negative values with large magnitudes of hundreds of meters per day (Figure 14b), i.e., an order of magnitude greater than what is attributed to mesoscale eddies alone [39]. Vertical velocities have a width of 5 to $10 \mathrm{~km}$. They are intensified in the ocean interior, below the mixed layer down to at least $500 \mathrm{~m}$. Large values of $w$ are collocated with strong buoyancy gradients, and are therefore mostly found on the edges of, and in between, eddies. The vertical velocity field is coherent over a few dives, suggesting that IGWs do not contribute to it. Around $1100 \mathrm{~km}$ for example, the signature of IGWs is noticeable on buoyancy gradients below the MLD (Figure 12b) but vertical velocities are weak (Figure 14b). This is consistent with the physics captured by the omega equation, in the sense that $\nabla b$ due to IGWs are not necessarily correlated with velocity gradients and hence do not contribute to the vertical velocity field derived from the omega equation [17]. In addition, IGWs are known to impact buoyancy gradients when $N / f \sim 300$ [40], which is not the case here as $N / f \sim 60$.
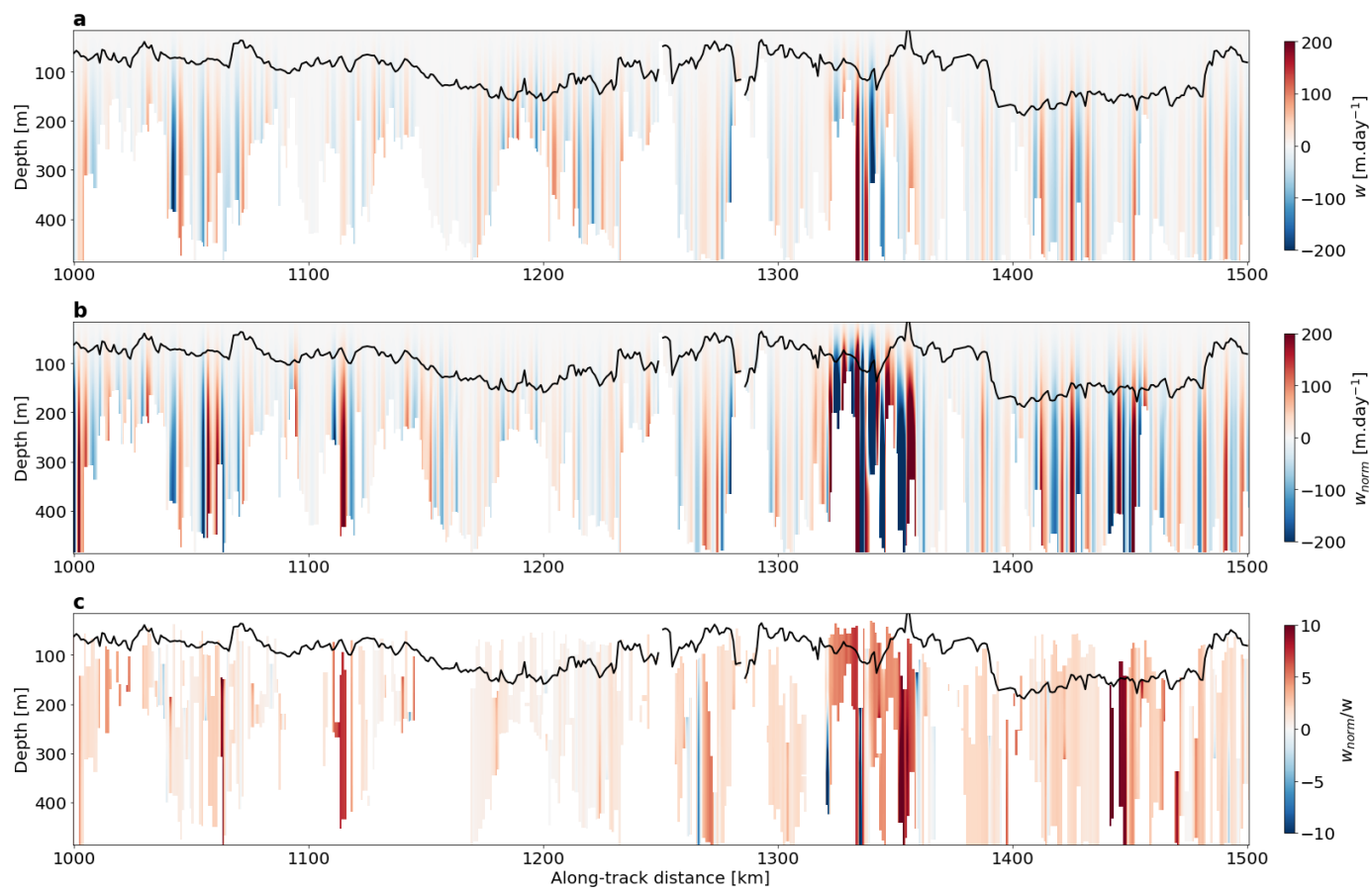

Figure 14. Vertical section of (a) vertical velocities $w$ along $x$ the curvilinear abscissa following the seal's trajectory (in color in Figure 11), (b) vertical velocities $w_{\text {norm }}$ in the across-front direction, (c) $w_{\text {norm }} / w$ for $|w|>5 \mathrm{~m} \mathrm{day}^{-1}$. Vertical velocities are derived by solving a 2-D QG omega equation (Equation (2)). The MLD is shown in black and is defined as the level of a $0.03 \mathrm{~kg} \mathrm{~m}^{-3}$ density increase from $15 \mathrm{~m}$ depth.

Note that compared to the Sawyer Eliassen equation [41], there is a tendency for $w$ diagnosed here to be underestimated by $\sim 1.4$ [3]. However, the validity of using the omega equation to reconstruct the vertical velocity field has been explored by numerous studies [42,43] (see in particular Figure 3 in Qiu et al. [43]) and a comparison with $w$ from the model is presented in the supplementary material 
of Siegelman et al. [3]. Applying the same methodology to a synthetic trajectory extracted from the model would also be valuable to pursue in future work.

\section{Discussion}

in situ CTD observations at high-resolution can now be acquired by numerous platforms, including ocean/sea glider, shipboard-ADCP, SeaSoar, saildrone or animal-borne instrument $[1-4,6]$. Resulting 2-D $(x-z)$ sections often reveal numerous sub-mesoscale fronts extending down to $\sim 500 \mathrm{~m}$, especially in high EKE regions, that are characterized by high Rossby and low Richardson numbers. It emphasizes their energetic dynamics, which includes vertical velocities. As such, it has been suggested that these fronts considerably impact oceanic vertical transport [6]. However, inferring their dynamics requires knowledge of the past evolution of the 3-D structure of the horizontal velocity field over a few days, which is rarely accessible from high-resolution in situ data. To meet this challenge, we use a numerical simulation that includes numerous deep-reaching fronts associated with Rossby and Richardson numbers of order one. Results demonstrate that it is possible to recover the correct orientation, magnitude and growth rate of $\nabla b$ observations at depth from FSLE's orientation at the surface. In turn, this enables the inference of deep-reaching frontal dynamics in terms of vertical velocities.

However, the present study solely considers a region of high EKE of the ACC [19] characterized by $N / f \sim 60$, in springtime. This area might not be characteristic of the entire ACC in terms of EKE and vertical stratification, nor of other seasons (e.g., in winter one can expect submesoscales to be more energetic [6,44]). As such, these findings call for extended analyses at different seasons and throughout the world oceans to explore the generic character of our results.

However, based on the present numerical results, we propose a two-step procedure for the analysis of existing sub-mesoscale-resolving dataset using satellite altimetry observations:

A1. We recover the orientation of sampled lateral buoyancy gradients, and subsequently their correct magnitude through a normalization scheme. This normalization takes into account the angle between the sampling platform and the front's orientation deduced from the FSLE's eigenvector.

A2. We diagnose vertical velocities from the normalized lateral buoyancy gradients using a 2-D QG version of the omega equation.

A weakness of step A1. is that it fails when the platform moves in the along-front direction. In this case, the sine of the angle between the front and the platform is close to 0 , which may lead to spurious normalized values. Furthermore, as mentioned in the main text, the QG omega equation used in step A2. underestimates vertical velocities (see supplementary information in Siegelman et al. [3] for a discussion).

Results of the present study can also be applied to the planning of in situ experiments, using satellite altimetry data in real time to design an optimal sampling strategy of sub-mesoscale ocean fronts. We would like to suggest the following strategy, intended for ship-board instruments or remote piloting of an ocean robot:

B1. Identify areas of strong background strain from near-real time satellite observations of geostrophic currents (i.e., when the Okubo-Weiss quantity is positive, Figure 11c).

B2. Use near-real time AVISO-FSLE products to identify the location and orientation of FSLE filaments (Figure 11d).

B3. Pilot the ship/robot perpendicular to this orientation. One should then perpendicularly cross sub-mesoscale fronts embedded in the background flow.

Note that even though mesoscale eddies evolve slowly compared to sub-mesoscale fronts, their geometry, and therefore stirring properties, can change on timescales of $O$ (day) as mentioned in Appendix A. As a result, FSLE vary quickly in time and their maxima can move from a quarter of a degree in just two days (see for example at $50.75^{\circ} \mathrm{S}$ and $51^{\circ} \mathrm{S}$ in Figure 15). As such, it is important 
to use near-real time FSLE distributed by AVISO in step B2. When possible, we encourage users to compute their own FSLE from near-real time SSH observations as near-real-time FSLE are provided by AVISO with a 20-day delay. In addition, while this method is viable for a ship that travels $\geq$ $200 \mathrm{~km} /$ day, it is not suitable for every instrument. For instance, gliders only travel $\sim 20 \mathrm{~km} /$ day so the features are evolving at scales that make it difficult to achieve this planning.
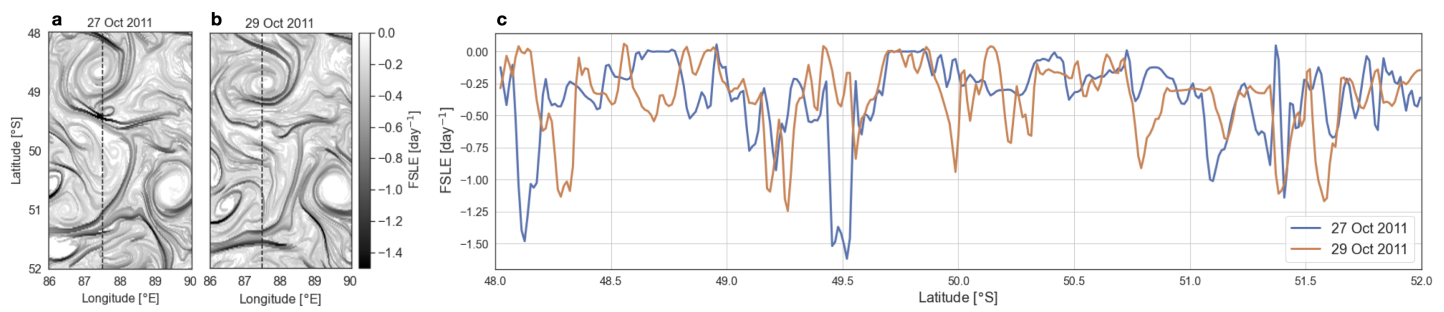

Figure 15. (a) FSLE $_{\text {filt }}$ at $39 \mathrm{~m}$ on 27 October 2011 00:00:00 UTC. (b) FSLE filt at $39 \mathrm{~m}$ on 29 October 2011 00:00:00 UTC. The dashed gray line at $87.5^{\circ}$ E corresponds to the section in (c). (c) Meridional profile of FSLE filt $_{\text {at }} 39 \mathrm{~m}$ and $87.5^{\circ} \mathrm{E}$ on 27 October 2011 00:00:00 UTC, 2011 (blue line) and 29 October 2011 00:00:00 UTC (orange line).

Step B3. accounts for the potential co-location mismatch between $\nabla b$ and FSLE, despite their common alignment. Indeed, the position of a large FSLE filament is not necessarily indicative of the presence of a deep sub-mesoscale front at this exact location, but rather, of the presence of one or several fronts in its vicinity, that are aligned with the FSLE's orientation (Figure 1c). As such, repeating long-enough transect perpendicular to the FSLE's orientation should enable the crossing of several sub-mesoscale fronts at an angle of $90^{\circ}$ (for example $\sim 50 \mathrm{~km}$-long transects separated by $\sim 10 \mathrm{~km}$ each). Furthermore, the sampling time should take into account the synoptic scale associated with the time evolution of the mesoscale field's geometry $(O($ day $))$, and ideally each transect should be collected in less than $\sim 10 \mathrm{~h}$. This method appears well suited for deep-reaching sub-mesoscale fronts that are elongated over tens of kilometers (a variant of which has been successfully used in Legal et al. [45]) but a potential drawback is that it may fail for shorter and smaller fronts, such as those present within the mixed layer.

Results of this study should gain increasing utility in the context of upcoming satellite missions, such as SWOT or WACM, that will provide high-resolution observations of the surface currents on a global scale. These future observations should help refine our results and eventually extend them to smaller scales. Given the recent technological advances in in situ sampling, sub-mesoscale-resolving datasets are expected to become increasingly available such that the approaches proposed here should have increasing utility (e.g., the upcoming S-MODE experiment).

Author Contributions: Methodology, L.S. and P.K.; formal analysis, L.S., P.K.; writing—original draft preparation, L.S., P.K. and A.F.T.; writing-review and editing, L.S., P.K., A.F.T., H.S.T., D.M.; numerical simulation, D.M., H.S.T. All authors have read and agreed to the published version of the manuscript.

Funding: L.S., H.S.T. and D.M. carried research at the Jet Propulsion Laboratory, California Institute of Technology, under a contract with NASA with support from the Physical Oceanography (PO) and Modeling, Analysis, and Prediction (MAP) Programs. L.S. is a NASA-JVSRP affiliate and is supported by a Caltech-JPL postdoctoral fellowship. P.K. is supported by a NASA NPP Senior Fellowship and by the SWOT and S-Mode projects. A.F.T. is supported by the David and Lucille Packard Foundation and NASA grant NNX16AG42G.

Acknowledgments: High-end computing resources for the numerical simulation were provided by the NASA Advanced Supercomputing (NAS) Division at the Ames Research Center. Thanks to Christopher Henze at NASA Ames Hyperwall and MITgcm developers and NAS scientists that made available the model outputs. This work was supported by the CNES-TOSCA project Elephant seals as Oceanographic Samplers of sub-mesoscale features led by C. Guinet with support of the French Polar Institute (Programs 109 and 1201). Thanks to Fabien Roquet and Baptiste Picard that made available the seal's data.

Conflicts of Interest: The authors declare no conflict of interest. 


\section{Appendix A. On the Growth Rate and Orientation of Buoyancy Gradient}

As mentioned in the main text, the time evolution of a buoyancy gradient is given by :

$$
\frac{d \nabla b}{d t}=-\mathbf{A} \nabla b-N^{2} \nabla w,
$$

with $\nabla b$ the lateral buoyancy gradient, $w$ the vertical velocity field, $N^{2}$ the vertical stratification and $\mathbf{A}$ the velocity gradient tensor, defined as:

$$
\mathbf{A} \equiv \frac{1}{2}\left(\begin{array}{cc}
\sigma_{n} & \sigma_{s}+\zeta \\
\sigma_{s}-\zeta & -\sigma_{n}
\end{array}\right),
$$

with $\zeta=\partial_{x} v-\partial_{y} u$ the relative vorticity, $\sigma_{n}=\partial_{x} u-\partial_{y} v$ the normal strain rate, and $\sigma_{s}=\partial_{x} v+\partial_{y} u$ the shear strain rate [29].

From Equation (A1), one can see that the growth of $\nabla b$ depends on the eigenvalues of $\mathbf{A}$. A commonly used criterion to infer the production of $\nabla b$ is the Okubo-Weiss quantity $(W)$, derived by Okubo [30] and Weiss [46,47]. $W$ is the squared eigenvalues of $\mathbf{A}$ :

$$
W=\frac{1}{4}\left(\sigma_{n}^{2}+\sigma_{s}^{2}-\zeta^{2}\right) .
$$

The Okubo-Weiss quantity is used in Section 3 to partition the fluid into elliptic regions dominated by $\zeta$, and hyperbolic regions dominated by $\sigma_{n}$ and $\sigma_{s}$. Hyperbolic regions coincide with an excess of the magnitude of strain over vorticity and conversely for elliptic ones. Under the assumption that the velocity gradient is slowly varying along a Lagrangian trajectory, the behavior of $\nabla b$ can be determined by $W:|\nabla b|$ do not grow in vortex cores where $W<0$ since the eigenvalues of $\mathbf{A}$ are purely imaginary. In this case, the gradient vector experiences a simple rotation. On the other hand, in strain-dominated areas where $W>0$, the eigenvalues of $\mathbf{A}$ are real and $|\nabla b|$ exponentially grow. Thus, strain-dominated areas are particularly prone to the formation of sub-mesoscale fronts. These mechanisms are further detailed in many studies [32,48-51] and we summarize some of them below.

The asymmetric part of $\mathbf{A}$ is explained by the relative vorticity whose effect is only to rotate $\nabla b$. The symmetric part is explained by the strain field whose effects are (i) to align $\nabla b$ toward the compressional strain direction (one of the eigenvectors of the symmetric part of $\mathbf{A}$ ) and (ii) to increase the magnitude of $\nabla b$ with a maximum growth rate equal to $\sigma=\sqrt{\sigma_{n}^{2}+\sigma_{s}^{2}}$. Using $\left(\sigma_{n}, \sigma_{s}\right)=$ $\sigma(\sin (2 \phi), \cos (2 \phi))$, the compressional strain direction has an angle with the $x$-axis equal to $\alpha=$ $-\phi-\pi / 4$ [32] (Figure A1). Using $\nabla b=|\nabla b|(\cos (\theta), \sin (\theta))$, with $\theta$ the angle between $\nabla b$ and the $x$-axis (Figure A1), and ignoring $w$, the growth and alignment properties of $\nabla b$ are given by [32]:

$$
\begin{gathered}
\frac{1}{|\nabla b|} \frac{d|\nabla b|}{d t}=\frac{\sigma}{2} \cos (2(\theta-\alpha)), \\
\frac{d(\theta-\alpha)}{d t}=\sigma\left(\frac{\zeta+d \phi / d t}{\sigma}-\sin (2(\theta-\alpha))\right),
\end{gathered}
$$

with $d \phi / d t$ the rotation of the strain axis along a Lagrangian trajectory, which has a typical magnitude of $\zeta$ [31,32]. Thus, from Equation (A4), the growth rate of $\nabla b$ is $\sigma \cos (2(\theta-\alpha))$ [32]. The typical time scale for $\theta-\alpha$ to reach a steady state is of the order of $\sigma^{-1}$ (i.e., $O($ day)) [32]. Equation (A5) means that in most cases, the orientation of $\nabla b(\theta)$ differs from the orientation of the strain compressional axis $(\alpha)$. The growth rate and orientation of tracer gradients given by Equations (A4) and (A5) have been validated using numerical simulations with high resolution [32,37]. 


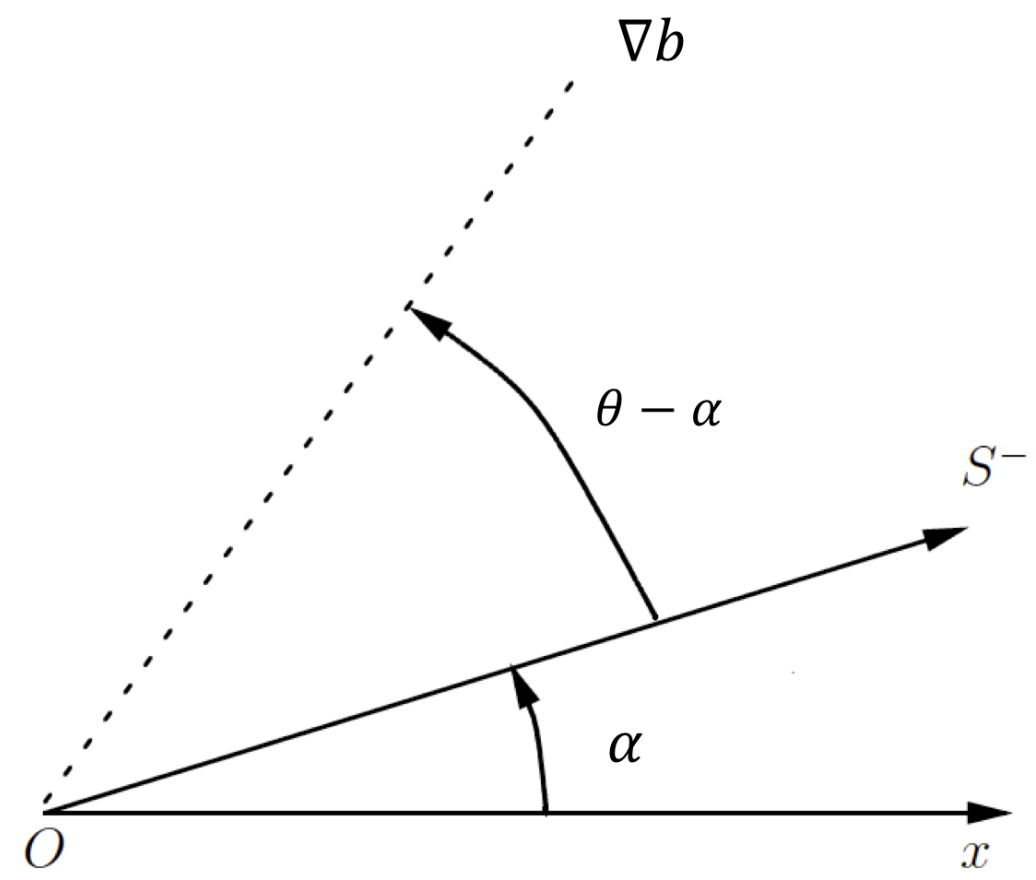

Figure A1. $\theta$ is the angle between $\nabla b$ and the $x$-axis and $\alpha$ is the angle between the compressional strain vector $\left(S^{-}\right)$and the $x$-axis.

Integrating Equation (A5) to get $\nabla b$-orientation requires knowledge of the time evolution of A. A simpler method to get $\theta$ is to estimate FSLE that implicitly use the same information for the velocity field [11-13]. Equation (A4) can then be used to estimate the growth rate of $\nabla b$ from satellite observations. Indeed, in addition to $\theta$ diagnosed from FSLE (using satellite observations), $\alpha$ and $\sigma$ can be retrieved from the strain components estimated from surface geostrophic currents (diagnosed from altimetry) via $\alpha=\frac{1}{2} \arctan \left(\frac{\sigma_{s}}{\sigma_{n}}\right)$ and $\sigma=\sqrt{\sigma_{n}^{2}+\sigma_{s}^{2}}$. However, the growth rate of $\nabla b$ can be further simplified if expressed in terms of the velocity field in the Cartesian frame associated with $\nabla b^{\prime}$ s orientation. Indeed, using the definition of $\sigma_{n}$ and $\sigma_{s}$, the growth rate in Equation (A4) can be written as:

$$
G R=\frac{\sigma}{2} \cos (2(\theta-\alpha))=-\frac{1}{2}\left(2 \partial_{x} u \cos 2 \theta+\left(\partial_{x} v+\partial_{y} u\right) \sin 2 \theta\right) .
$$

with GR the growth rate. Then, when the Cartesian frame of reference, as well as the velocity field, are rotated by $\theta$, the growth rate becomes:

$$
G R=-u_{x} \text { norm }
$$

with $u_{\text {norm }}$ the cross-front horizontal velocity, $x$ the cross-front direction and $u_{x \text { norm }}$ (i.e., $\partial_{x} u_{\text {norm }}$ ) the cross-front velocity gradient. The growth rate given by Equation (A7) is the one used in Section 4 (Equation (7)). Note that a more accurate estimation of the growth rate has been proposed by previous studies [31,52]. This estimation is based on the Lagrangian acceleration that drive the time evolution of the velocity field [31] and, consequently, explicitly takes into account the time evolution of the strain field $\sigma$. However, because of the assumptions considered in this study and in particular the use of only a 2-D quasi-geostrophic Omega equation, we have chosen the simplest estimation of the growth rate, i.e., the one given by Equations (A6) and (A7). 


\section{Appendix B. Frequency-Wavenumber Spectrum}

We refer the reader to [9] for the methodology used to compute $\omega-k$ spectrum.Figure A2 shows $\omega-k$ spectra of KE, strain rate and $|\nabla b|$ at $39 \mathrm{~m}, 99 \mathrm{~m}$ and $506 \mathrm{~m}$ computed in the study area from 27 October to 27 December 2011. They have a similar shape from the surface down to $506 \mathrm{~m}$. As expected, most of the KE is contained at mesoscale $(>50 \mathrm{~km}$ ) and low frequencies (Figure A2a-c). The relative contribution of IGWs is weak compared to that of balanced motions. The strain rate and $|\nabla b|$ exhibit a different distribution with a large part of the variance contained at submesoscales $(<50 \mathrm{~km}$, Figure A2d-i). Above $506 \mathrm{~m}$, strain rate and $|\nabla b|$ are only weakly affected by linear IGWs (above the dashed line), whereas at $506 \mathrm{~m}$ the impact of linear IGWs is proportionally greater. Overall, the $\omega$ - $k$ spectra indicate that the contribution of submesoscales $(\leq 50 \mathrm{~km})$ to the strain rate and to $\nabla b$ is significant and that linear IGWs have a weak impact away from the seasonal and permanent thermocline. These results emphasize the existence of energetic submesoscales in the ocean interior.
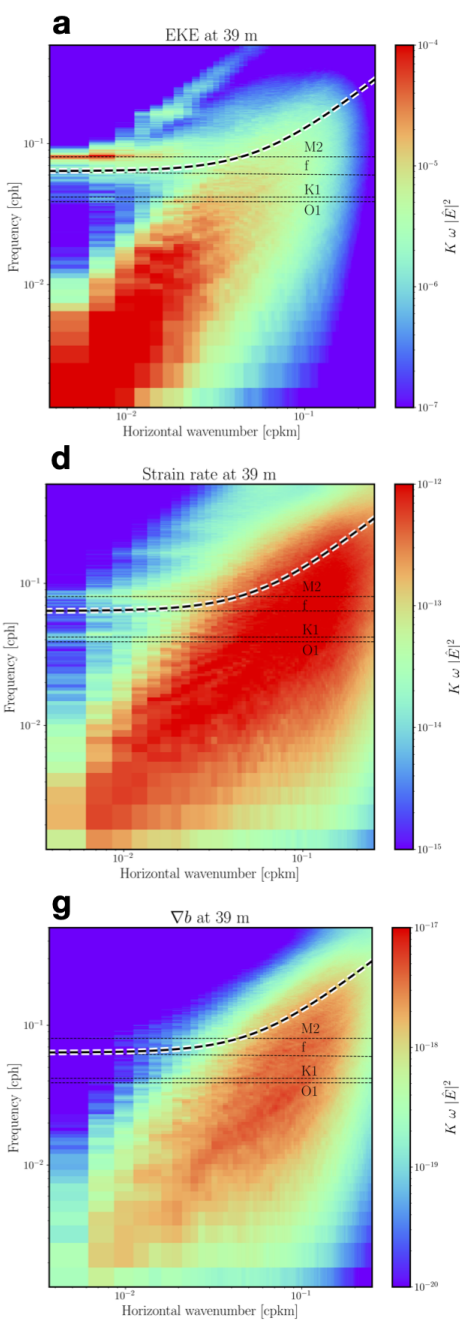

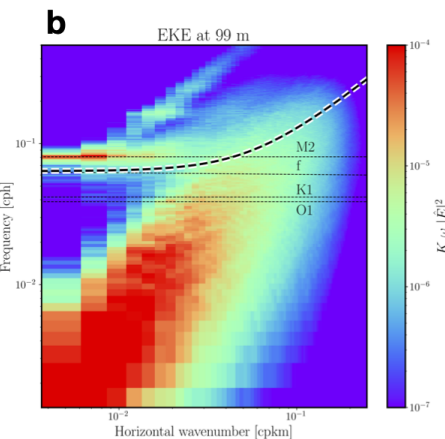

e

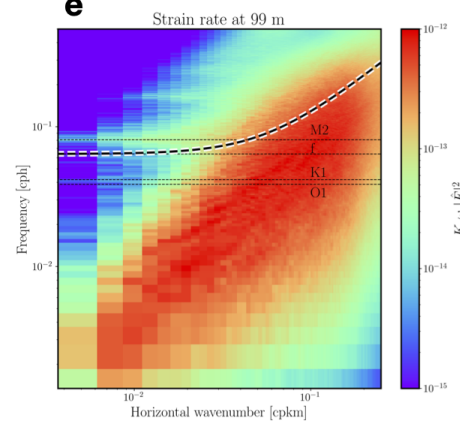

h

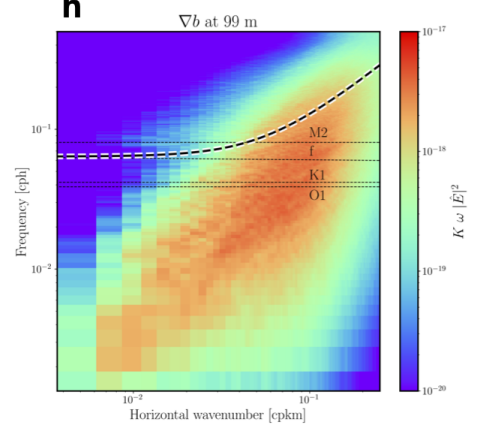

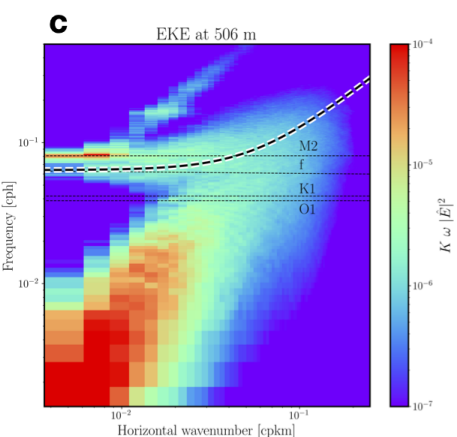

$\mathbf{f}$
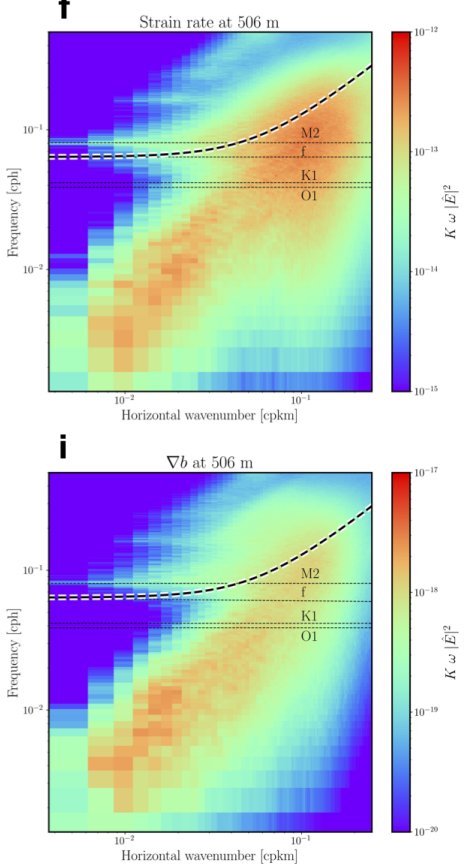

Figure A2. Frequency-wavenumber spectra computed from October 27 to December 27, 2011 of (a) KE at $39 \mathrm{~m},(\mathbf{b}) \mathrm{KE}$ at $99 \mathrm{~m}$, (c) KE at $506 \mathrm{~m}$, (d) Strain rate at $39 \mathrm{~m}$, (e) Strain rate at $99 \mathrm{~m}$, (f) Strain rate at $506 \mathrm{~m},(\mathrm{~g})|\nabla b|$ at $39 \mathrm{~m},(\mathbf{h})|\nabla b|$ at $99 \mathrm{~m},(\mathbf{i})|\nabla b|$ at $506 \mathrm{~m}$. The dashed line corresponds to the dispersion relation curve (associated with linear internal gravity waves, IGWs) that corresponds to the highest baroclinic mode resolved by the simulation. This curve allows for the partition between balanced motions (below the curve) and IGWs (above the curve). These spectra are presented in a variance preserving form, which allows the direct comparison of the relative contribution of different time and spatial scales to the total variance. 


\section{Appendix C. Filter Cutoff Wavelength}

A 2-D Gaussian smoother filter is applied isotropically to the model horizontal velocities field with a cutoff wavelength $\lambda_{c}=20 \mathrm{~km}$. This filter is thoroughly described in Appendix C of Chelton et al. [53]. However, briefly, the 2-D weighting function for isotropic smoothing can be expressed as

$$
w^{2 d}(x, y)=w^{2 d}(r)
$$

where $r=\left(x^{2}+y^{2}\right)^{1 / 2}$ is the radial distance from the estimation location to each data point within the 2-D span of the smoother. Here, we use a Gaussian-weighted smoother for which the weighting function in one dimension is

$$
w_{G}(x)=\frac{1}{\sqrt{\pi} L_{G}} e^{-x^{2} / L_{G}^{2}},
$$

where $L_{G}$ defines the e-folding scale of Gaussian weighting. The normalization factor $\sqrt{\pi} L_{G}$ constrains the weighting function (A9) to have unit area, as required for unbiased smoothing. The parameter $L_{G}$ of the Gaussian smoother (A9) is related to the desired half-power filter cutoff wavenumber $k_{c}$ and associated wavelength $\lambda_{c}=k_{c}^{-1}$ by

$$
L_{G}=\left(-\frac{\ln (0.5)}{2 \pi^{2}}\right)^{1 / 2} \frac{1}{k_{c}}=0.187 \lambda_{c} .
$$

\section{References}

1. Thompson, A.F.; Lazar, A.; Buckingham, C.; Naveira Garabato, A.C.; Damerell, G.M.; Heywood, K.J. Open-ocean submesoscale motions: A full seasonal cycle of mixed layer instabilities from gliders. J. Phys. Oceanogr. 2016, 46, 1285-1307. [CrossRef]

2. Yu, X.; Naveira Garabato, A.C.; Martin, A.P.; Buckingham, C.E.; Brannigan, L.; Su, Z. An annual cycle of submesoscale vertical flow and restratification in the upper ocean. J. Phys. Oceanogr. 2019, 49, 1439-1461. [CrossRef]

3. Siegelman, L.; Klein, P.; Rivière, P.; Thompson, A.F.; Torres, H.S.; Flexas, M.; Menemenlis, D. Enhanced upward heat transport at deep submesoscale ocean fronts. Nat. Geosci. 2020, 13, 50-55. [CrossRef]

4. Siegelman, L. Energetic submesoscale dynamics in the ocean interior. J. Phys. Oceanogr. 2020, 50, 727-749. [CrossRef]

5. Thomas, L.N.; Tandon, A.; Mahadevan, A. Submesoscale processes and dynamics. Ocean. Model. Eddying Regime 2008, 177, 17-38.

6. Su, Z.; Wang, J.; Klein, P.; Thompson, A.F.; Menemenlis, D. Ocean submesoscales as a key component of the global heat budget. Nat. Commun. 2018, 9, 775. [CrossRef]

7. Marshall, J.; Adcroft, A.; Hill, C.; Perelman, L.; Heisey, C. A finite-volume, incompressible Navier Stokes model for studies of the ocean on parallel computers. J. Geophys. Res. Ocean. 1997, 102, 5753-5766. [CrossRef]

8. Hill, C.; Menemenlis, D.; Ciotti, B.; Henze, C. Investigating solution convergence in a global ocean model using a 2048-processor cluster of distributed shared memory machines. Sci. Program. 2007, 15, 107-115. [CrossRef]

9. Torres, H.S.; Klein, P.; Menemenlis, D.; Qiu, B.; Su, Z.; Wang, J.; Chen, S.; Fu, L.L. Partitioning ocean motions into balanced motions and internal gravity waves: A modeling study in anticipation of future space missions. J. Geophys. Res. Ocean. 2018, 123, 8084-8105. [CrossRef]

10. d'Ovidio, F.; Fernández, V.; Hernández-García, E.; López, C. Mixing structures in the Mediterranean Sea from finite-size Lyapunov exponents. Geophys. Res. Lett. 2004, 31. [CrossRef] [CrossRef]

11. Lapeyre, G. Characterization of finite-time Lyapunov exponents and vectors in two-dimensional turbulence. Chaos Interdiscip. J. Nonlinear Sci. 2002, 12, 688-698. [CrossRef] [PubMed]

12. Keating, S.R.; Smith, K.S.; Kramer, P.R. Diagnosing lateral mixing in the upper ocean with virtual tracers: Spatial and temporal resolution dependence. J. Phys. Oceanogr. 2011, 41, 1512-1534. [CrossRef] 
13. Waugh, D.W.; Abraham, E.R. Stirring in the global surface ocean. Geophys. Res. Lett. 2008, 35. [CrossRef] [CrossRef]

14. Siegelman, L.; Roquet, F.; Mensah, V.; Rivière, P.; Pauthenet, E.; Picard, B.; Guinet, C. Correction and accuracy of high-and low-resolution ctd data from animal-borne instruments. J. Atmos. Ocean. Technol. 2019, 36, 745-760. [CrossRef]

15. Intergovernmental-Oceanographic-Commission. The International Thermodynamic Equation of Seawater-2010: Calculation and Use of Thermodynamic Properties. [Includes Corrections Up to 31st October 2015]; UNESCO: Paris, France, 2010.

16. Hoskins, B.J.; Draghici, I.; Davies, H.C. A new look at the $\omega$-equation. Q. J. R. Meteorol. Soc. 1978, 104, 31-38. [CrossRef]

17. Giordani, H.; Prieur, L.; Caniaux, G. Advanced insights into sources of vertical velocity in the ocean. Ocean. Dyn. 2006, 56, 513-524. [CrossRef]

18. Burns, K.J.; Vasil, G.M.; Oishi, J.S.; Lecoanet, D.; Brown, B. Dedalus: Dedalus: A flexible framework for numerical simulations with spectral methods. Phys. Rev. Res. 2020, 2, 023068. [CrossRef]

19. Thompson, A.F.; Naveira Garabato, A.C. Equilibration of the Antarctic Circumpolar Current by standing meanders. J. Phys. Oceanogr. 2014, 44, 1811-1828. [CrossRef]

20. Vallis, G.K. Atmospheric and Oceanic Fluid Dynamics; Cambridge University Press: Cambridge, UK, 2017.

21. Haynes, P.; Anglade, J. The vertical-scale cascade in atmospheric tracers due to large-scale differential advection. J. Atmos. Sci. 1997, 54, 1121-1136. [CrossRef]

22. Klein, P.; Treguier, A.M.; Hua, B.L. Three-dimensional stirring of thermohaline fronts. J. Mar. Res. 1998, 56, 589-612. [CrossRef]

23. Meunier, T.; Ménesguen, C.; Schopp, R.; Le Gentil, S. Tracer stirring around a meddy: The formation of layering. J. Phys. Oceanogr. 2015, 45, 407-423. [CrossRef]

24. Hua, B.L.; Haidvogel, D.B. Numerical simulations of the vertical structure of quasi-geostrophic turbulence. J. Atmos. Sci. 1986, 43, 2923-2936. [CrossRef]

25. Smith, K.S.; Ferrari, R. The production and dissipation of compensated thermohaline variance by mesoscale stirring. J. Phys. Oceanogr. 2009, 39, 2477-2501. [CrossRef]

26. Molemaker, M.J.; McWilliams, J.C.; Capet, X. Balanced and unbalanced routes to dissipation in an equilibrated Eady flow. J. Fluid Mech. 2010, 654, 35-63. [CrossRef]

27. Roullet, G.; Klein, P. Cyclone-anticyclone asymmetry in geophysical turbulence. Phys. Rev. Lett. 2010, 104, 218501. [CrossRef] [PubMed]

28. Klein, P.; Lapeyre, G.; Siegelman, L.; Qiu, B.; Fu, L.L.; Torres, H.; Su, Z.; Menemenlis, D.; Le Gentil, S. Ocean-Scale Interactions From Space. Earth Space Sci. 2019, 6, 795-817. [CrossRef]

29. Lapeyre, G.; Klein, P.; Hua, B.L. Oceanic Restratification Forced by Surface Frontogenesis. J. Phys. Oceanogr. 2006, 36, 1577-1590. [CrossRef]

30. Okubo, A. Horizontal dispersion of floatable particles in the vicinity of velocity singularities such as convergences. Deep. Sea Res. Oceanogr. Abstr. 1970, 17, 445-454. [CrossRef]

31. Hua, B.L.; Klein, P. An exact criterion for the stirring properties of nearly two-dimensional turbulence. Phys. D Nonlinear Phenom. 1998, 113, 98-110. [CrossRef]

32. Lapeyre, G.; Klein, P.; Hua, B.L. Does the tracer gradient vector align with the strain eigenvectors in 2D turbulence? Phys. Fluids 1999, 11, 3729-3737. [CrossRef]

33. Smith, K.S.; Vallis, G.K. The scales and equilibration of midocean eddies: Freely evolving flow. J. Phys. Oceanogr. 2001, 31, 554-571. [CrossRef]

34. Lapeyre, G. What vertical mode does the altimeter reflect? On the decomposition in baroclinic modes and on a surface-trapped mode. J. Phys. Oceanogr. 2009, 39, 2857-2874. [CrossRef]

35. Foussard, A.; Berti, S.; Perrot, X.; Lapeyre, G. Relative dispersion in generalized two-dimensional turbulence. J. Fluid Mech. 2017, 821, 358-383. [CrossRef]

36. Scott, R. Local and nonlocal advection of a passive scalar. Phys. Fluids 2006, 18, 116601. [CrossRef]

37. Lapeyre, G.; Hua, B.L.; Klein, P. Dynamics of the orientation of active and passive scalars in two-dimensional turbulence. Phys. Fluids 2001, 13, 251-264. [CrossRef]

38. Ballarotta, M.; Ubelmann, C.; Pujol, M.I.; Taburet, G.; Fournier, F.; Legeais, J.F.; Faugère, Y.; Delepoulle, A.; Chelton, D.; Dibarboure, G.; et al. On the resolutions of ocean altimetry maps. Ocean. Sci. 2019, 15, 1091-1109. [CrossRef] 
39. McGillicuddy, D.J., Jr. Mechanisms of physical-biological-biogeochemical interaction at the oceanic mesoscale. Annu. Rev. Mar. Sci. 2016, 8, 125-159. [CrossRef]

40. Kunze, E. Near-inertial wave propagation in geostrophic shear. J. Phys. Oceanogr. 1985, 15, 544-565. [CrossRef]

41. Hakim, G.; Keyser, D. Canonical frontal circulation patterns in terms of Green's functions for the Sawyer-Eliassen equation. Q. J. R. Meteorol. Soc. 2001, 127, 1795-1814. [CrossRef]

42. Viúdez, Á.; Tintoré, J.; Haney, R.L. About the nature of the generalized omega equation. J. Atmos. Sci. 1996, 53, 787-795. [CrossRef]

43. Qiu, B.; Chen, S.; Klein, P.; Torres, H.; Wang, J.; Fu, L.L.; Menemenlis, D. Reconstructing Upper-Ocean Vertical Velocity Field from Sea Surface Height in the Presence of Unbalanced Motion. J. Phys. Oceanogr. 2020, 50, 55-79. [CrossRef]

44. Sasaki, H.; Klein, P.; Qiu, B.; Sasai, Y. Impact of oceanic-scale interactions on the seasonal modulation of ocean dynamics by the atmosphere. Nat. Commun. 2014, 5, 6636. [CrossRef] [PubMed]

45. Legal, C.; Klein, P.; Tréguier, A.M.; Paillet, J. Diagnosis of the vertical motions in a mesoscale stirring region. J. Phys. Oceanogr. 2007, 37, 1413-1424. [CrossRef]

46. Weiss, J. The dynamics of enstrophy transfer in two-dimensional hydrodynamics. Phys. D Nonlinear Phenom. 1991, 48, 273-294. [CrossRef]

47. Weiss, J. Report LJI-TN-81-121; La Jolla Inst.: San Diego, CA, USA, 1981.

48. Gent, P.R.; McWilliams, J.C. Consistent balanced models in bounded and periodic domains. Dyn. Atmos. Ocean. 1983, 7, 67-93. [CrossRef]

49. Basdevant, C.; Philipovitch, T. On the validity of the "Weiss criterion" in two-dimensional turbulence. Phys. D Nonlinear Phenom. 1994, 73, 17-30. [CrossRef]

50. Hua, B.L.; McWilliams, J.C.; Klein, P. Lagrangian accelerations in geostrophic turbulence. J. Fluid Mech. 1998, 366, 87-108. [CrossRef]

51. Muraki, D.J.; Snyder, C.; Rotunno, R. The next-order corrections to quasigeostrophic theory. J. Atmos. Sci. 1999, 56, 1547-1560. [CrossRef]

52. Klein, P.; Hua, B.L.; Lapeyre, G. Alignment of tracer gradient vectors in 2D turbulence. Phys. D Nonlinear Phenom. 2000, 146, 246-260. [CrossRef]

53. Chelton, D.B.; Schlax, M.G.; Samelson, R.M.; Farrar, J.T.; Molemaker, M.J.; McWilliams, J.C.; Gula, J. Prospects for future satellite estimation of small-scale variability of ocean surface velocity and vorticity. Prog. Oceanogr. 2019, 173, 256-350. [CrossRef] 